\title{
X\#Ray and Optical Variability in NGC 4051 and the Nature of Narrow\#Line Seyfert 1 Galaxies
}

\section{Citation}

Peterson, B. M., I. M. McHardy, B. J. Wilkes, P. Berlind, R. Bertram, M. Calkins, S. J. Collier, et al. 2000. "X\#Ray and Optical Variability in NGC 4051 and the Nature of Narrow\#Line Seyfert 1 Galaxies." The Astrophysical Journal 542 (1) (October 10): 161-174. doi:10.1086/309518.

\section{Published Version}

doi:10.1086/309518

\section{Permanent link}

http://nrs.harvard.edu/urn-3:HUL.InstRepos:30212192

\section{Terms of Use}

This article was downloaded from Harvard University's DASH repository, and is made available under the terms and conditions applicable to Other Posted Material, as set forth at http:// nrs.harvard.edu/urn-3:HUL.InstRepos:dash.current.terms-of-use\#LAA

\section{Share Your Story}

The Harvard community has made this article openly available.

Please share how this access benefits you. Submit a story.

\section{Accessibility}


THE ASTROPHYSICAL JOURNAL, 542:161-174, 2000 October 10

(c) 2000. The American Astronomical Society. All rights reserved. Printed in U.S.A.

\title{
X-RAY AND OPTICAL VARIABILITY IN NGC 4051 AND THE NATURE OF NARROW-LINE SEYFERT 1 GALAXIES
}

\author{
B. M. Peterson,${ }^{1}$ I. M. McHardy, ${ }^{2}$ B. J. Wilkes,${ }^{3}$ P. Berlind,,${ }^{3}$ R. Bertram,${ }^{1,4}$ M. Calkins,${ }^{3}$ S. J. Collier,${ }^{1}$ \\ J. P. Huchra, ${ }^{3}$ S. Mathur, ${ }^{1}$ I. Papadakis, ${ }^{5}$ J. Peters, ${ }^{3,6}$ R. W. Pogge, ${ }^{1}$ P. Romano, ${ }^{1}$ S. Tokarz, ${ }^{3}$ \\ P. UtTley, ${ }^{2}$ M. VestergaARD, ${ }^{1}$ AND R. M. WAGNeR ${ }^{1,4}$ \\ Received 2000 January 27; accepted 2000 May 19
}

\begin{abstract}
We report on the results of a three-year program of coordinated X-ray and optical monitoring of the narrow-line Seyfert 1 galaxy NGC 4051. The rapid continuum variations observed in the X-ray spectra are not detected in the optical, although the time-averaged X-ray and optical continuum fluxes are well correlated. Variations in the flux of the broad $\mathrm{H} \beta$ line are found to lag behind the optical continuum variations by 6 days (with an uncertainty of 2-3 days), and combining this with the line width yields a virial mass estimate of $\sim 1.1 \times 10^{6} M_{\odot}$, at the very low end of the distribution of active galactic nucleus masses measured by line reverberation. Strong variability of He II $\lambda 4686$ is also detected, and the response time measured is similar to that of $\mathrm{H} \beta$ but with a much larger uncertainty. The He II $\lambda 4686$ line is almost 5 times broader than $\mathrm{H} \beta$, and it is strongly blueward asymmetric, as are the high-ionization UV lines recorded in archival spectra of NGC 4051. The data are consistent with the Balmer lines arising in a low-to-moderate-inclination disklike configuration and the high-ionization lines arising in an outflowing wind, of which we observe preferentially the near side. Previous observations of the narrowline region morphology of this source suggest that the system is inclined by $\sim 50^{\circ}$, and if this is applicable to the broad $\mathrm{H} \beta$-emitting region, a central mass of $\sim 1.4 \times 10^{6} M_{\odot}$ can be inferred. During the third year of monitoring, both the X-ray continuum and the He II $\lambda 4686$ line went into extremely low states, although the optical continuum and the $\mathrm{H} \beta$ broad line were both still present and variable. We suggest that the inner part of the accretion disk may have gone into an advection-dominated state, yielding little radiation from the hotter inner disk.
\end{abstract}

Subject headings: galaxies: active — galaxies: individual (NGC 4051) - galaxies: nuclei galaxies: Seyfert - X-rays: galaxies

\section{INTRODUCTION}

The broad components of permitted emission lines in the spectra of active galactic nuclei (AGNs) typically have velocity widths of a few to several thousand kilometers per second. The defining characteristic of the subclass of AGNs known as narrow-line Seyfert 1 galaxies (NLS1s) is that the broad components of their emission lines are much narrower $\left(V_{\mathrm{FWHM}} \lesssim 2000 \mathrm{~km} \mathrm{~s}^{-1}\right)$ than is typical for broad-line objects (Osterbrock \& Pogge 1985). NLS1s are extreme AGNs in other respects as well: their UV-optical properties correlate well with the Boroson \& Green (1992) primary spectral eigenvector identified in principal component analysis. In other words, NLS1 classification correlates well with strong optical Fe II emission and weak [O III] $\lambda \lambda 4959$,

\footnotetext{
${ }^{1}$ Department of Astronomy, Ohio State University, 140 West 18th Avenue, Columbus, OH 43210; peterson@astronomy.ohio-state.edu, stefan@astronomy.ohio-state.edu, smita@astronomy.ohio-state.edu, pogge@astronomy.ohio-state.edu, promano@astronomy.ohio-state.edu, vester@astronomy.ohio-state.edu.

${ }^{2}$ Department of Physics and Astronomy, University of Southampton, Southampton SO17 1BJ, England, UK; imh@astro.soton.ac.uk, pu@astro.soton.ac.uk.

${ }^{3}$ Harvard-Smithsonian Center for Astrophysics, 60 Garden Street, Cambridge, MA 02138; belinda@cfa.harvard.edu, pberlind@ cfa.harvard.edu, mcalkins@cfa.harvard.edu, huchra@cfa.harvard.edu, stokarz@cfa.harvard.edu.

${ }^{4}$ Steward Observatory, University of Arizona, Tucson, AZ 85721; rayb@as.arizona.edu,rmw@as.arizona.edu.

${ }^{5}$ Department of Physics, University of Crete, P.O. Box 2208, 71003 Heraklion, Crete, Greece; jhep@physics.uoc.gr.

${ }^{6}$ Deceased.
}

5007 emission (Boller, Brandt, \& Fink 1996). While the possible importance of such extreme AGNs has been recognized for two decades (Davidson \& Kinman 1978), interest in NLS1s has increased recently as their unusual X-ray properties have come to light: they have unusually steep soft and hard X-ray spectra (Puchnarewicz et al. 1992; Boller et al. 1996; Brandt, Mathur, \& Elvis 1997) and undergo rapid nonlinear variability (Boller et al. 1997). While rapid, large-amplitude variability in the UV-optical has not been reported, existing data do not address well the relationship between the X-ray and long-wavelength variations. A good compilation of the observed properties of NLS1s is given by Taniguchi, Murayama, \& Nagao (2000).

Possible explanations for the narrowness of the permitted lines include the following:

1. NLS1s have more distant than normal broad-line regions (BLRs) (Mason, Puchnarewicz, \& Jones 1996; Wandel \& Boller 1998). The line widths are attributed to virial motion $\left(M \approx V^{2} r / G\right)$ in the vicinity of a supermassive black hole, but the orbital velocities are smaller than in more typical AGNs because of greater distance to the central source.

2. NLS1s are low-inclination (i.e., nearly face-on) systems (Osterbrock \& Pogge 1985). In this model, the line widths are again due to orbital motion around the central black hole, and the bulk of the BLR gas orbits in a common plane that is almost perpendicular to the line of sight, leading to relatively small Doppler widths.

3. NLS1s have relatively low black hole masses but high accretion rates. Again, the basic assumption is that the BLR 
motions are virial, but the central source has a lower mass. The luminosity can be kept relatively high by supposing that the accretion rate (relative to the Eddington rate) is correspondingly high in these sources.

The third of these explanations forms the current paradigm for NLS1s, as it explains not only the narrow emission lines but possibly also the steepness of the soft X-ray spectrum: the temperature structure of an optically thick, geometrically thin accretion disk is given by

$$
T(r)=6.3 \times 10^{5}\left(\dot{M} / \dot{M}_{\mathrm{Edd}}\right)^{1 / 4} M_{8}^{-1 / 4}\left(r / R_{\mathrm{S}}\right)^{-3 / 4} \mathrm{~K},
$$

where $M_{8}$ is the black hole mass in units of $10^{8} M_{\odot}$ and $R_{\mathrm{S}}$ is the Schwarzschild radius (Shakura \& Sunyaev 1973). The temperature of the inner regions of the disk scales as $M^{-1 / 4}$, so the strength of the soft X-rays in NLS1s might plausibly be ascribed to a low central mass and a high accretion rate flow.

One way to distinguish among various explanations of the NLS1 phenomenon is to measure the size of the BLR, which can be done via reverberation-mapping techniques (Blandford \& McKee 1982; Peterson 1993; Netzer \& Peterson 1997). It is usually assumed that the variability of an emission line $L(t)$ to a variable continuum $C(t)$ can be linearized to the form

$$
\delta L(t)=\int \Psi(\tau) \delta C(t-\tau) d \tau,
$$

where $\Psi(\tau)$ is the "transfer function," which depends on the geometry and reprocessing physics of the BLR. A representative timescale for response of a line can be found by a simple cross-correlation of the continuum and emission-line light curves. By convolving equation (2) with $C(t)$, one obtains the cross-correlation function

$$
F_{\mathrm{CCF}}(\tau)=\int \Psi\left(\tau^{\prime}\right) F_{\mathrm{ACF}}\left(\tau-\tau^{\prime}\right) d \tau^{\prime},
$$

where $F_{\mathrm{ACF}}(\tau)$ is the continuum autocorrelation function (Penston 1991; Peterson 1993). The cross-correlation lag $\tau$ can be taken to be the light-travel time across the BLR, so the BLR size is given by $r=c \tau$. By combining this with the emission-line width $V_{\text {FWHM }}$, the mass of the central source can be inferred to be

$$
M=\frac{f V_{\mathrm{FWHM}}^{2} c \tau}{G},
$$

where $f$ is a factor of order unity that depends on the still unknown geometry and kinematics of the BLR. There is an implicit assumption that the gravitational force of the central object dominates the kinematics of the BLR; this is formally unproven, but, at least in the case of the wellstudied Seyfert 1 galaxy NGC 5548, the reverberationmapping data are consistent with the required $V_{\text {FWHM }} \propto$ $r^{-1 / 2}$ relationship (Peterson \& Wandel 1999). Virial mass estimates based on reverberation-mapping data are now available for nearly 40 AGNs (Wandel, Peterson, \& Malkan 1999; Kaspi et al. 2000).

Beginning in early 1996, we undertook a program of contemporaneous X-ray and optical spectroscopic monitoring of the galaxy NGC 4051, the only NLS1 galaxy in Seyfert's (1943) original list of high surface brightness galaxies with strong emission lines. The X-ray variability characteristics of NGC 4051 are typical of the NLS1 class (Lawrence et al. 1987; McHardy et al. 1995).

X-ray observations were made with the Rossi $X$-Ray Timing Explorer (RXTE), and optical spectra were obtained with ground-based telescopes, as described below. The purpose of this program has been twofold:

1. To determine the nature of the relationship between the X-ray and UV-optical continuum variations. This is a particularly interesting question in the case of NGC 4051, since the X-ray flux dropped to an extremely low level in 1998 May (Uttley et al. 1999), toward the end of this campaign.

2. To determine the BLR size and virial mass via reverberation techniques.

In this contribution, we present the results of this program and discuss their implications for the nature of the NLS1 phenomenon.

\section{OBSERVATIONS AND DATA ANALYSIS}

\subsection{X-Ray Observations}

The X-ray observations were made with the large area $\left(0.7 \mathrm{~m}^{2}\right)$ Proportional Counter Array (PCA) on $R X T E$ (Bradt, Rothschild, \& Swank 1993). The observations shown here, which are part of a continuing monitoring program, cover the period from 1996 March to 1998 June. The observations were scheduled initially to cover the largest range of variability timescales with the smallest number of observations in order to determine the X-ray power spectrum efficiently. The program therefore consisted of observations approximately every 7 to 10 days throughout the first year, followed by observations approximately every 2 weeks thereafter. In addition, during the first year, there were two more intensive monitoring periods: a 2 week period of twice-daily observations and a 4 week period of daily observations. Each observation lasted for typically $1 \mathrm{ks}$. In 1996 December, there was also a period of 3 days during which NGC 4051 was observed for a total of $70 \mathrm{ks}$.

The PCA consists of five proportional counter units (PCUs), but typically only three PCUs (numbers 0,1 , and 2) were operational, and all count rates refer to the total counts from those three PCUs. Where other than three PCUs were in operation, the count rate has been normalized to three PCUs. The PCUs have three layers. Here we only include data from the upper layer, as this layer provides the highest signal-to-noise ratio for photons in the energy range $2-20 \mathrm{keV}$ where the flux from NGC 4051 is strongest. We used standard "good time interval" (GTI) criteria to select data with the lowest background. We reject data obtained when the Earth elevation angle was less than $10^{\circ}$, when the pointing offset from the source was greater than 0.02 , or during passage through the South Atlantic Anomaly or up to 5 minutes afterward. The PCA is a nonimaging device with a field of view of FWHM $\sim 1^{\circ}$, and so the background that we subtract must be calculated from a model. Here we use the FTOOL routine PCABACKEST V2.0c, with the new "L7" model, to calculate the background.

The resultant $2-10 \mathrm{keV}$ light curve is shown in the top panel of Figure 1. Further details of the X-ray light curves and variability are given by I. E. Papadakis et al. (2000, in preparation). For NGC 4051, 10 counts $\mathrm{s}^{-1}(2-10 \mathrm{keV})$, 


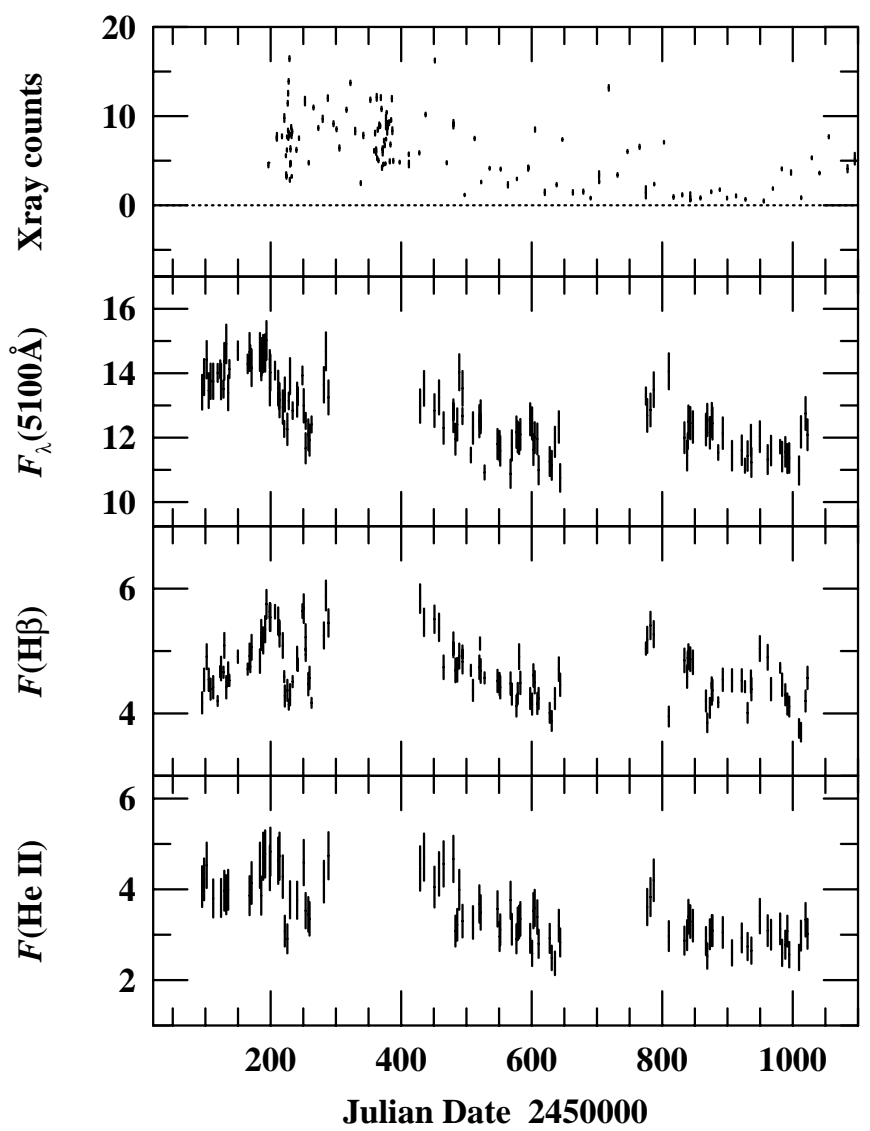

FIG. 1.-Continuum and emission-line light curves for NGC 4051 between 1996 January and 1998 June. The top panel shows the X-ray light curve for NGC 4051 in counts $\mathrm{s}^{-1}$ over the $2-10 \mathrm{keV}$ bandpass; 1 count $\mathrm{s}^{-1}$ corresponds to a flux of approximately $10^{-12} \mathrm{ergs} \mathrm{cm}^{-2} \mathrm{~s}^{-1}$ over the $2-10 \mathrm{keV}$ range. The second panel shows the optical continuum light curve in units of $10^{-15} \mathrm{ergs} \mathrm{s}^{-1} \mathrm{~cm}^{-2} \AA^{-1}$, as in Table 2 . The third and fourth panels show, respectively, the $\mathrm{H} \beta$ and $\mathrm{He}$ II $\lambda 4686$ emission-line light curves, in units of $10^{-13} \mathrm{ergs} \mathrm{s}^{-1} \mathrm{~cm}^{-2}$, also as in Table 2.

from three PCUs, corresponds to a flux of $4 \times 10^{-11}$ ergs $\mathrm{cm}^{-2} \mathrm{~s}^{-1}$.

\subsection{Optical Spectroscopy}

Optical spectroscopic observations were obtained between UT 1996 January 12 (JD 2,450,095) and 1998 July 28 (JD 2,451,022), covering three separate observing seasons. Observations were made with the Ohio State CCD Spectrograph on the $1.8 \mathrm{~m}$ Perkins Telescope of the Ohio State and Ohio Wesleyan Universities at the Lowell Observatory (data set A) and with the FAST spectrograph (Fabricant et al. 1998) on the $1.5 \mathrm{~m}$ Tillinghast Reflector of the Center for Astrophysics on Mount Hopkins (data set B). A log of these observations is presented in Table 1. Column (1) gives the UT date of each observation, and the Julian Date is given in column (2). The origin of the data (set A or B) appears in column (3). The projected size of the spectrograph entrance aperture was $5^{\prime \prime} .0 \times 7.5$ (i.e., a slit width of 5."0 and a cross-dispersion extraction window of 7".5) for all set A spectra and 3".0 $\times$ 4".6 for all set B spectra. In each case, the slit was oriented in the east-west direction (i.e., the slit position angle was always $90^{\circ}$ ). The nominal spectral resolution was $9 \AA$ for all set A spectra and $5 \AA$ for all set B
TABLE 1

LOG OF SPECTROSCOPIC OBSERVATIONS

\begin{tabular}{|c|c|c|c|c|}
\hline $\begin{array}{l}\text { UT Date } \\
\text { (1) }\end{array}$ & $\begin{array}{c}\text { Julian Date } \\
(-2,450,000) \\
(2)\end{array}$ & $\begin{array}{l}\text { Code } \\
\text { (3) }\end{array}$ & $\begin{array}{l}\text { Range } \\
(\AA ̊) \\
(4)\end{array}$ & $\begin{array}{l}\text { File Name } \\
\text { (5) }\end{array}$ \\
\hline 1996 Jan 12 . & 95.0 & B & $3670-7450$ & $\mathrm{n} 00095 \mathrm{~b}$ \\
\hline 1996 Jan 15. & 98.0 & B & $3650-7520$ & n00098b \\
\hline 1996 Jan 19 ....... & 102.0 & B & $3650-7410$ & $\mathrm{n} 00102 \mathrm{~b}$ \\
\hline 1996 Jan $22 \ldots \ldots \ldots$ & 105.1 & B & $3660-7410$ & $\mathrm{n} 00105 \mathrm{~b}$ \\
\hline 1996 Jan 25 ....... & 108.0 & B & $3650-7520$ & n00108b \\
\hline 1996 Jan 29. & 112.0 & B & $3640-7500$ & $\mathrm{n} 00112 \mathrm{~b}$ \\
\hline $1996 \mathrm{Feb} 05$ & 118.8 & A & $4510-5670$ & $\mathrm{n} 00118 \mathrm{a}$ \\
\hline 1996 Feb 09 & 122.8 & A & $4520-5670$ & $\mathrm{n} 00122 \mathrm{a}$ \\
\hline 1996 Feb 10 & 124.0 & B & $3660-7400$ & $\mathrm{n} 00123 \mathrm{~b}$ \\
\hline 1996 Feb 14 & 127.8 & A & $4520-5670$ & $\mathrm{n} 00127 \mathrm{a}$ \\
\hline 1996 Feb 15 & 129.0 & B & $3660-7420$ & $\mathrm{n} 00129 \mathrm{~b}$ \\
\hline 1996 Feb 18 & 132.0 & B & $3660-7530$ & $\mathrm{n} 00132 \mathrm{~b}$ \\
\hline $1996 \mathrm{Feb} 21 \ldots \ldots$ & 134.9 & B & $3660-7410$ & n00134b \\
\hline 1996 Feb 23 & 136.8 & A & $4520-5680$ & n00136a \\
\hline 1996 Mar $07 \ldots$ & 149.8 & A & $4520-5680$ & n00149a \\
\hline $1996 \operatorname{Mar} 22 \ldots$ & 164.8 & A & $4530-5680$ & $\mathrm{n} 00164 \mathrm{a}$ \\
\hline $1996 \operatorname{Mar} 25 \ldots \ldots$ & 167.7 & B & $3640-7500$ & $\mathrm{n} 00167 \mathrm{~b}$ \\
\hline 1996 Mar $26 \ldots \ldots$ & 168.8 & A & $4520-5690$ & $\mathrm{n} 00168 \mathrm{a}$ \\
\hline 1996 Mar $28 \ldots \ldots$ & 170.8 & B & $3650-7390$ & $\mathrm{n} 00170 \mathrm{~b}$ \\
\hline 1996 Apr 10 ...... & 183.6 & B & $3660-7420$ & n00183b \\
\hline 1996 Apr 12 & 185.6 & B & $3670-7420$ & $\mathrm{n} 00185 \mathrm{~b}$ \\
\hline 1996 Apr 12 & 185.7 & A & $4540-5690$ & $\mathrm{n} 00185 \mathrm{a}$ \\
\hline 1996 Apr 15 & 188.6 & B & $3660-7410$ & $\mathrm{n} 00188 \mathrm{~b}$ \\
\hline 1996 Apr 18 & 191.6 & B & $3660-7410$ & n00191b \\
\hline 1996 Apr 20 & 193.6 & B & $3660-7400$ & n00193b \\
\hline 1996 Apr 25 & 198.7 & A & $4510-5680$ & $\mathrm{n} 00198 \mathrm{a}$ \\
\hline 1996 Apr 25 & 198.8 & B & $3650-7410$ & n00198b \\
\hline 1996 Apr 26 & 199.6 & B & $3660-7400$ & n00199b \\
\hline 1996 May $03 . . . \ldots$. & 206.7 & A & $4530-5690$ & n00206a \\
\hline 1996 Мay $08 . . . .$. & 211.6 & B & $3660-7400$ & $\mathrm{n} 00211 \mathrm{~b}$ \\
\hline 1996 Мay $09 . \ldots \ldots$ & 212.8 & A & $4540-5680$ & $\mathrm{n} 00212 \mathrm{a}$ \\
\hline 1996 May $10 \ldots \ldots$ & 213.6 & B & $3660-7520$ & $\mathrm{n} 00213 \mathrm{~b}$ \\
\hline 1996 May $15 \ldots \ldots$ & 218.6 & B & $3660-7510$ & n00218b \\
\hline 1996 May $17 \ldots \ldots$ & 220.8 & A & $4540-5700$ & n00220a \\
\hline 1996 May $18 \ldots \ldots$ & 221.7 & B & $3660-7400$ & n00221b \\
\hline 1996 May $22 \ldots . .$. & 225.6 & B & $3670-7430$ & $\mathrm{n} 00225 \mathrm{~b}$ \\
\hline 1996 May $24 \ldots . .$. & 227.8 & A & $4540-5700$ & $\mathrm{n} 00227 \mathrm{a}$ \\
\hline 1996 Мay $26 . . . .$. & 229.7 & B & $3670-7430$ & $\mathrm{n} 00229 \mathrm{~b}$ \\
\hline 1996 May $30 \ldots \ldots$ & 233.7 & A & $4530-5670$ & n00233a \\
\hline 1996 Jun $06 \ldots . .$. & 240.6 & B & $3660-7400$ & $\mathrm{n} 00240 \mathrm{~b}$ \\
\hline 1996 Jun $07 . \ldots \ldots$ & 241.7 & A & $4530-5690$ & n00241a \\
\hline 1996 Jun $14 \ldots \ldots$. & 248.7 & A & $4540-5690$ & n00248a \\
\hline 1996 Jun $16 . \ldots \ldots$. & 250.7 & B & $3600-7540$ & $\mathrm{n} 00250 \mathrm{~b}$ \\
\hline 1996 Jun 19 ....... & 253.6 & B & $3670-7530$ & $\mathrm{n} 00253 \mathrm{~b}$ \\
\hline 1996 Jun $19 . \ldots \ldots$. & 253.7 & A & $4520-5680$ & $\mathrm{n} 00253 \mathrm{a}$ \\
\hline 1996 Jun $23 \ldots \ldots \ldots$ & 257.7 & B & $3660-7420$ & $\mathrm{n} 00257 \mathrm{~b}$ \\
\hline 1996 Jun $25 \ldots \ldots \ldots$ & 259.6 & B & $3670-7550$ & $\mathrm{n} 00259 \mathrm{~b}$ \\
\hline 1996 Jun 28 ....... & 262.7 & A & $4520-5680$ & $\mathrm{n} 00262 \mathrm{a}$ \\
\hline 1996 Jul $17 \ldots \ldots \ldots$ & 281.6 & B & $3670-7520$ & n00281b \\
\hline 1996 Jul $20 \ldots \ldots \ldots$ & 284.7 & B & $3670-7530$ & n00284b \\
\hline 1996 Jul $24 \ldots \ldots \ldots$ & 288.6 & B & $3670-7530$ & $\mathrm{n} 00288 \mathrm{~b}$ \\
\hline 1996 Dec $11 \ldots \ldots$ & 429.0 & B & $3650-7510$ & n00429b \\
\hline 1996 Dec $17 \ldots \ldots$ & 435.0 & B & $3660-7520$ & $\mathrm{n} 00435 \mathrm{~b}$ \\
\hline 1997 Jan 02 ....... & 451.0 & B & $3670-7510$ & n00451b \\
\hline 1997 Jan 09 ....... & 458.0 & B & $3670-7510$ & $\mathrm{n} 00458 \mathrm{~b}$ \\
\hline 1997 Jan 16 ....... & 465.0 & B & $4000-7500$ & $\mathrm{n} 00465 \mathrm{~b}$ \\
\hline 1997 Jan $31 \ldots \ldots$. & 479.9 & A & $4720-5990$ & n00479a \\
\hline 1997 Jan $31 \ldots \ldots$. & 480.0 & B & $4000-7500$ & $\mathrm{n} 00480 \mathrm{~b}$ \\
\hline 1997 Feb 03 ...... & 483.1 & B & $4000-7500$ & n00483b \\
\hline 1997 Feb 06 ...... & 485.9 & B & $4000-7530$ & $\mathrm{n} 00485 \mathrm{~b}$ \\
\hline 1997 Feb 09 ...... & 489.0 & B & $4000-7500$ & n00488b \\
\hline 1997 Feb $14 \ldots \ldots$ & 493.9 & A & $4380-5550$ & n00493a \\
\hline 1997 Feb $14 \ldots \ldots$ & 494.0 & B & $4000-7520$ & n00494b \\
\hline $1997 \mathrm{Feb} 27 \ldots \ldots$ & 506.8 & A & $4360-5500$ & $\mathrm{n} 00506 \mathrm{a}$ \\
\hline 1997 Mar $02 \ldots \ldots$. & 510.0 & B & $3660-7520$ & $\mathrm{n} 00509 \mathrm{~b}$ \\
\hline 1997 Mar $12 \ldots \ldots$ & 519.8 & B & $3660-7500$ & $\mathrm{n} 00519 \mathrm{~b}$ \\
\hline 1997 Mar $13 . \ldots \ldots$ & 520.8 & A & $4290-5820$ & $\mathrm{n} 00520 \mathrm{a}$ \\
\hline
\end{tabular}


TABLE $1-$ Continued

\begin{tabular}{|c|c|c|c|c|}
\hline $\begin{array}{l}\text { UT Date } \\
\text { (1) }\end{array}$ & $\begin{array}{c}\text { Julian Date } \\
(-2,450,000) \\
(2)\end{array}$ & $\begin{array}{l}\text { Code } \\
\text { (3) }\end{array}$ & $\begin{array}{l}\text { Range } \\
(\AA ̊) \\
(4)\end{array}$ & $\begin{array}{c}\text { File Name } \\
\text { (5) }\end{array}$ \\
\hline 1997 Mar 14. & 521.8 & B & $3670-7310$ & n00521b \\
\hline 1997 Mar 20 & 527.8 & A & $4390-5940$ & $\mathrm{n} 00527 \mathrm{a}$ \\
\hline 1997 Apr 09 & 547.6 & B & $3670-7500$ & $\mathrm{n} 00547 \mathrm{~b}$ \\
\hline 1997 Apr 12 & 550.8 & B & $3670-7520$ & $\mathrm{n} 00550 \mathrm{~b}$ \\
\hline 1997 Apr 13 & 551.8 & B & $3680-7540$ & n00551b \\
\hline 1997 Apr 29. & 567.6 & B & $3670-7530$ & $\mathrm{n} 00567 \mathrm{~b}$ \\
\hline 1997 May $01 \ldots . .$. & 569.6 & B & $3670-7540$ & $\mathrm{n} 00569 \mathrm{~b}$ \\
\hline 1997 May 08. & 576.6 & B & $3650-7500$ & $\mathrm{n} 00576 \mathrm{~b}$ \\
\hline 1997 May $10 \ldots . .$. & 578.6 & B & $3650-7520$ & $\mathrm{n} 00578 \mathrm{~b}$ \\
\hline 1997 May $12 .$. & 580.7 & B & $3660-7520$ & $\mathrm{n} 00580 \mathrm{~b}$ \\
\hline 1997 May $14 \ldots \ldots$ & 582.6 & B & $3650-7530$ & $\mathrm{n} 00582 \mathrm{~b}$ \\
\hline 1997 Маy 29. & 597.7 & B & $3660-7520$ & n00597b \\
\hline 1997 Jun $01 \ldots$ & 600.6 & B & $4000-7530$ & $\mathrm{n} 00600 \mathrm{~b}$ \\
\hline 1997 Jun $03 \ldots \ldots$. & 602.6 & B & $3660-7540$ & $\mathrm{n} 00602 \mathrm{~b}$ \\
\hline 1997 Jun $05 .$. & 604.6 & B & $3670-7530$ & $\mathrm{n} 00604 \mathrm{~b}$ \\
\hline 1997 Jun $09 \ldots$ & 608.6 & B & $3670-7530$ & $\mathrm{n} 00608 \mathrm{~b}$ \\
\hline 1997 Jun 11 .. & 610.6 & B & $3670-7540$ & $\mathrm{n} 00610 \mathrm{~b}$ \\
\hline 1997 Jun 28 .. & 627.6 & B & $3670-7530$ & $\mathrm{n} 00627 \mathrm{~b}$ \\
\hline 1997 Jul $01 .$. & 630.7 & B & $3670-7520$ & $\mathrm{n} 00630 \mathrm{~b}$ \\
\hline 1997 Jul $06 \ldots$ & 635.6 & B & $3670-7530$ & $\mathrm{n} 00635 \mathrm{~b}$ \\
\hline 1997 Jul $12 \ldots$ & 641.6 & B & $3670-7530$ & $\mathrm{n} 00641 \mathrm{~b}$ \\
\hline 1997 Jul $14 \ldots$ & 643.6 & B & $3670-7520$ & $\mathrm{n} 00643 \mathrm{~b}$ \\
\hline 1997 Nov 22. & 775.0 & A & $4310-5800$ & $\mathrm{n} 00775 \mathrm{a}$ \\
\hline 1997 Nov 24. & 777.0 & B & 3750 & $\mathrm{n} 00777 \mathrm{~b}$ \\
\hline 1997 Nov 29 ...... & 782.0 & B & $3750-7500$ & $\mathrm{n} 00782 \mathrm{~b}$ \\
\hline 1997 Dec 04 & 787.0 & B & $3750-7500$ & $\mathrm{n} 00787 \mathrm{~b}$ \\
\hline 1997 Dec $27 \ldots \ldots$ & 810.1 & B & $3670-7530$ & $\mathrm{n} 00810 \mathrm{~b}$ \\
\hline 1998 Jan $20 \ldots . .$. & 834.0 & B & $3680-7540$ & $\mathrm{n} 00834 \mathrm{~b}$ \\
\hline 1998 Jan $24 \ldots \ldots$. & 838.1 & B & $3660-7550$ & $\mathrm{n} 00838 \mathrm{~b}$ \\
\hline 1998 Jan 25 .. & 839.0 & A & $4330-5840$ & $\mathrm{n} 00839 \mathrm{a}$ \\
\hline 1998 Jan $26 \ldots$ & 840.1 & B & $3670-7540$ & $\mathrm{n} 00840 \mathrm{~b}$ \\
\hline 1998 Jan $29 .$. & 843.0 & B & $3650-7520$ & $\mathrm{n} 00843 \mathrm{~b}$ \\
\hline 1998 Feb 02 & 847.0 & B & $3660-7540$ & $\mathrm{n} 00847 \mathrm{~b}$ \\
\hline 1998 Feb 22 & 867.0 & B & $3650-7530$ & n00867b \\
\hline 1998 Feb $24 \ldots \ldots$ & 869.0 & B & $3670-7540$ & $\mathrm{n} 00869 \mathrm{~b}$ \\
\hline 1998 Feb 28 & 873.0 & B & $3670-7530$ & $\mathrm{n} 00872 \mathrm{~b}$ \\
\hline 1998 Mar 03 ..... & 875.8 & A & $4300-5830$ & $\mathrm{n} 00876 \mathrm{a}$ \\
\hline 1998 Mar $03 \ldots \ldots$ & 876.0 & B & $3660-7530$ & $\mathrm{n} 00876 \mathrm{~b}$ \\
\hline 1998 Mar $04 \ldots . .$. & 877.0 & B & 366 & $\mathrm{n} 00877 \mathrm{~b}$ \\
\hline 1998 Mar $13 \ldots \ldots$ & 885.8 & A & $4380-5910$ & n00886a \\
\hline 1998 Mar $20 \ldots . .$. & 892.8 & B & $3660-7510$ & $\mathrm{n} 00892 \mathrm{~b}$ \\
\hline 1998 Apr 03 ...... & 906.9 & B & $3630-7490$ & $\mathrm{n} 00906 \mathrm{~b}$ \\
\hline 1998 Apr $18 \ldots \ldots$ & 921.9 & B & $3610-7470$ & $\mathrm{n} 00921 \mathrm{~b}$ \\
\hline 1998 Apr $23 \ldots \ldots$ & 926.8 & $\mathrm{~A}$ & $4320-5840$ & $\mathrm{n} 00927 \mathrm{a}$ \\
\hline 1998 Apr $27 \ldots \ldots$ & 930.8 & B & $3620-7500$ & n00930b \\
\hline 1998 May $02 \ldots . .$. & 935.9 & B & $3630-7490$ & $\mathrm{n} 00935 \mathrm{~b}$ \\
\hline 1998 May $03 . . . .$. & 936.6 & B & $3620-7490$ & n00936b \\
\hline 1998 May $16 \ldots \ldots$ & 949.6 & B & $3670-7510$ & n00949b \\
\hline 1998 May $28 \ldots . .$. & 961.6 & B & $3660-7530$ & n00961b \\
\hline 1998 Jun $02 \ldots \ldots$. & 966.7 & B & $3660-7540$ & n00966b \\
\hline 1998 Jun $16 \ldots . .$. & 980.7 & A & $4310-5850$ & $\mathrm{n} 00981 \mathrm{a}$ \\
\hline 1998 Jun $16 \ldots \ldots \ldots$ & 980.7 & B & $3660-7540$ & n00980b \\
\hline 1998 Jun $19 . \ldots \ldots$ & 983.7 & B & $3650-7520$ & n00983b \\
\hline 1998 Jun $24 \ldots . .$. & 988.6 & B & $3680-7530$ & $\mathrm{n} 00988 \mathrm{~b}$ \\
\hline 1998 Jun $27 \ldots \ldots$. & 991.7 & B & $3660-7530$ & n00991b \\
\hline 1998 Jun $30 \ldots \ldots$. & 994.6 & B & $3680-7520$ & $\mathrm{n} 00994 \mathrm{~b}$ \\
\hline 1998 Jul $15 \ldots \ldots \ldots$ & 1009.6 & B & $3710-7510$ & $\mathrm{n} 01009 \mathrm{~b}$ \\
\hline 1998 Jul $18 \ldots \ldots \ldots$ & 1012.6 & B & $3670-7530$ & $\mathrm{n} 01012 \mathrm{~b}$ \\
\hline 1998 Jul $25 \ldots \ldots \ldots$ & 1019.6 & B & $3700-7500$ & $\mathrm{n} 01019 \mathrm{~b}$ \\
\hline 1998 Jul $28 \ldots \ldots \ldots$ & 1022.6 & B & $3670-7530$ & $\mathrm{n} 01022 \mathrm{~b}$ \\
\hline
\end{tabular}

Note.-Codes for data origin: $\mathrm{A}=1.8 \mathrm{~m}$ Perkins Telescope + Ohio State CCD Spectrograph; $B=1.5 \mathrm{~m}$ Tillinghast Reflector + FAST Spectrograph. spectra. The wavelength coverage of each spectrum is given in column (4), and the file name is given in column (5). All of these spectra are publicly available on the International AGN Watch site on the World Wide Web. ${ }^{7}$

The spectroscopic images were processed in standard fashion for CCD frames, including bias subtraction, darkcount correction when necessary, flat-field correction, wavelength calibration, and flux calibration based on standard-star observations. Since even under photometric conditions AGN spectrophotometry is rarely more accurate than $\sim 10 \%$, the usual technique of flux calibration by comparison with standard stars is far too poor for AGN variability studies. We therefore base our flux calibration on a scale defined by the observed flux in the prominent narrow [O III] $\lambda \lambda 4959,5007$ doublet. These lines originate in a low-density region that is more spatially extended than the BLR or the continuum source, which for practical purposes can be regarded as point sources. The larger light-travel time and long recombination time ensure that any narrowline variations will occur only over much longer timescales than are of interest in this experiment. We therefore assume that the [O III] lines are constant in flux and use these to scale each spectrum. All spectra are scaled to a constant flux of $F([\mathrm{O} \mathrm{III}] \lambda 5007)=(3.91 \pm 0.12) \times 10^{-13} \mathrm{ergs} \mathrm{s}^{-1} \mathrm{~cm}^{-2}$, which is based on the mean of 10 spectra from data set $A$ that were obtained under photometric observing conditions during the 1996 observing season. The [O III] $\lambda 5007$ flux measured from photometric spectra from subsequent years supports our assumption that this value can be assumed to be constant over the timescales of interest. All of the spectra are adjusted in flux to have this value of $F([\mathrm{O} \mathrm{III}] \lambda 5007)$ by employing the spectral scaling software described by van Groningen \& Wanders (1992). The process is as follows: spectra are adjusted in flux by a multiplicative constant that is determined by comparing each spectrum to a "reference spectrum" that has been formed by averaging all of the highest quality (i.e., typically signal-to-noise ratios $\mathrm{S} / \mathrm{N} \gtrsim$ 30) spectra and scaling this mean spectrum to the adopted [O III] $\lambda 5007$ flux. All individual spectra are scaled relative to the reference spectrum in a least-squares fashion that minimizes the $[\mathrm{O}$ III] residuals in the difference spectrum produced by subtracting the reference spectrum from each individual spectrum. This program also corrects for small zero-point wavelength calibration errors between the individual spectra and takes resolution differences into account.

At this point, measurements of each of the spectra are made. The continuum flux at $\sim 5100 \AA$ (in the rest frame of NGC 4051, $z=0.002418$, based on $21 \mathrm{~cm}$ emission; de Vaucouleurs et al. 1991) is determined by averaging the flux in the 5090-5120 $\AA$ bandpass (in the observed frame). The $\mathrm{H} \beta$ emission-line flux is measured by assuming a linear underlying continuum between $\sim 4772$ and $\sim 5105 \AA$ and integrating the flux above this continuum between 4820 and $4910 \AA$ (all wavelengths in the observed frame). The longwavelength cutoff of this integration band is chosen to avoid the Fe II contamination underneath [O III] 24959 . We also note that no attempt has been made to correct for contamination of the line measurement by the narrow-line

\footnotetext{
${ }^{7}$ The light curves and spectra are available at http:// www.astronomy.ohio-state.edu/ agnwatch. All publicly available International AGN Watch data can be accessed at this site, which also includes complete references to published AGN Watch papers.
} 
TABLE 2

Light CuRves

\begin{tabular}{|c|c|c|c|}
\hline $\begin{array}{c}\text { Julian Date } \\
(-2,450,000) \\
(1)\end{array}$ & $\begin{array}{c}F_{\lambda}(5100 \AA) \\
\left(10^{-15} \operatorname{ergs~s}^{-1} \mathrm{~cm}^{-2} \AA^{-1}\right) \\
(2)\end{array}$ & $\begin{array}{c}F(\mathrm{H} \beta) \\
\left(10^{-13} \mathrm{ergs} \mathrm{s}^{-1} \mathrm{~cm}^{-2}\right) \\
(3)\end{array}$ & $\begin{array}{c}F(\mathrm{He} \mathrm{II} \lambda 4686) \\
\left(10^{-13} \mathrm{ergs} \mathrm{s}^{-1} \mathrm{~cm}^{-2}\right) \\
(4)\end{array}$ \\
\hline $95.0 \ldots \ldots \ldots$ & $13.41 \pm 0.54$ & $4.17 \pm 0.17$ & $4.06 \pm 0.45$ \\
\hline $98.0 \ldots \ldots \ldots$ & $13.88 \pm 0.56$ & $4.54 \pm 0.18$ & $4.22 \pm 0.46$ \\
\hline $102.0 \ldots \ldots \ldots$ & $14.42 \pm 0.58$ & $4.91 \pm 0.20$ & $4.53 \pm 0.50$ \\
\hline $105.1 \ldots \ldots \ldots$ & $13.46 \pm 0.54$ & $4.54 \pm 0.18$ & $\ldots$ \\
\hline $108.0 \ldots \ldots \ldots$ & $13.75 \pm 0.55$ & $4.39 \pm 0.17$ & $\ldots$ \\
\hline $112.0 \ldots \ldots \ldots$ & $13.75 \pm 0.55$ & $4.42 \pm 0.18$ & $3.80 \pm 0.42$ \\
\hline $118.8 \ldots \ldots \ldots$ & $14.01 \pm 0.28$ & $4.20 \pm 0.08$ & $\ldots$ \\
\hline $122.8 \ldots \ldots \ldots$ & $14.12 \pm 0.28$ & $4.67 \pm 0.09$ & $\ldots$ \\
\hline $124.0 \ldots \ldots \ldots$ & $13.74 \pm 0.55$ & $4.72 \pm 0.19$ & $3.81 \pm 0.42$ \\
\hline $127.8 \ldots \ldots \ldots$ & $13.51 \pm 0.27$ & $4.66 \pm 0.09$ & $\ldots$ \\
\hline $129.0 \ldots \ldots \ldots$ & $14.37 \pm 0.57$ & $5.09 \pm 0.20$ & $3.97 \pm 0.44$ \\
\hline $132.0 \ldots \ldots \ldots$ & $14.91 \pm 0.60$ & $4.42 \pm 0.18$ & $3.88 \pm 0.43$ \\
\hline $134.9 \ldots \ldots \ldots$ & $13.39 \pm 0.54$ & $4.63 \pm 0.19$ & $3.99 \pm 0.44$ \\
\hline $136.8 \ldots \ldots \ldots$ & $14.13 \pm 0.28$ & $4.53 \pm 0.09$ & $\ldots$ \\
\hline $149.8 \ldots \ldots \ldots$ & $14.70 \pm 0.29$ & $4.91 \pm 0.10$ & $\ldots$ \\
\hline $164.8 \ldots \ldots \ldots$ & $14.29 \pm 0.29$ & $4.71 \pm 0.09$ & $\ldots$ \\
\hline $167.7 \ldots \ldots \ldots$ & $14.66 \pm 0.59$ & $4.92 \pm 0.20$ & $3.86 \pm 0.43$ \\
\hline $168.8 \ldots \ldots \ldots$ & $14.58 \pm 0.29$ & $4.75 \pm 0.09$ & $\ldots$ \\
\hline $170.8 \ldots \ldots \ldots$ & $14.17 \pm 0.57$ & $5.06 \pm 0.20$ & $4.14 \pm 0.46$ \\
\hline $183.6 \ldots \ldots \ldots$ & $14.66 \pm 0.59$ & $4.84 \pm 0.19$ & $4.53 \pm 0.50$ \\
\hline $185.6 \ldots \ldots \ldots$ & $14.51 \pm 0.58$ & $5.29 \pm 0.21$ & $3.86 \pm 0.42$ \\
\hline $185.7 \ldots \ldots \ldots$ & $14.06 \pm 0.28$ & $5.12 \pm 0.10$ & $\ldots$ \\
\hline $188.6 \ldots \ldots \ldots$ & $14.61 \pm 0.58$ & $5.17 \pm 0.21$ & $4.73 \pm 0.52$ \\
\hline $191.6 \ldots \ldots \ldots$ & $14.63 \pm 0.58$ & $5.35 \pm 0.21$ & $4.78 \pm 0.52$ \\
\hline $193.6 \ldots \ldots \ldots$ & $15.02 \pm 0.60$ & $5.75 \pm 0.23$ & $\ldots$ \\
\hline $198.7 \ldots \ldots \ldots$ & $14.43 \pm 0.29$ & $5.63 \pm 0.11$ & $\cdots$ \\
\hline $198.8 \ldots \ldots \ldots$ & $13.54 \pm 0.54$ & $5.55 \pm 0.22$ & $4.47 \pm 0.49$ \\
\hline $199.6 \ldots \ldots \ldots$ & $14.02 \pm 0.56$ & $5.54 \pm 0.22$ & $4.83 \pm 0.53$ \\
\hline $206.7 \ldots \ldots \ldots$ & $14.08 \pm 0.28$ & $5.63 \pm 0.11$ & $\ldots$ \\
\hline $211.6 \ldots \ldots \ldots$ & $13.54 \pm 0.54$ & $5.48 \pm 0.22$ & $4.63 \pm 0.51$ \\
\hline $212.8 \ldots \ldots \ldots$ & $13.15 \pm 0.26$ & $5.36 \pm 0.11$ & $\ldots$ \\
\hline $213.6 \ldots \ldots \ldots$ & $13.16 \pm 0.53$ & $5.28 \pm 0.21$ & $4.73 \pm 0.52$ \\
\hline $218.6 \ldots \ldots \ldots$ & $12.95 \pm 0.52$ & $5.09 \pm 0.20$ & $4.28 \pm 0.47$ \\
\hline $220.8 \ldots \ldots \ldots$ & $12.27 \pm 0.25$ & $4.59 \pm 0.09$ & $\ldots$ \\
\hline $221.7 \ldots \ldots \ldots$ & $13.34 \pm 0.53$ & $4.28 \pm 0.17$ & $3.08 \pm 0.34$ \\
\hline $225.6 \ldots \ldots \ldots$ & $12.26 \pm 0.49$ & $4.37 \pm 0.17$ & $2.91 \pm 0.32$ \\
\hline $227.8 \ldots \ldots \ldots$ & $13.14 \pm 0.26$ & $4.14 \pm 0.08$ & $\ldots$ \\
\hline $229.7 \ldots \ldots \ldots$ & $13.91 \pm 0.56$ & $4.30 \pm 0.17$ & $3.77 \pm 0.41$ \\
\hline $233.7 \ldots \ldots \ldots$ & $12.84 \pm 0.26$ & $4.51 \pm 0.09$ & $\ldots$ \\
\hline $240.6 \ldots \ldots \ldots$ & $13.18 \pm 0.53$ & $4.88 \pm 0.19$ & $3.76 \pm 0.41$ \\
\hline $241.7 \ldots \ldots \ldots$ & $13.29 \pm 0.27$ & $4.78 \pm 0.10$ & $\ldots$ \\
\hline $248.7 \ldots \ldots \ldots$ & $13.93 \pm 0.28$ & $5.64 \pm 0.11$ & $\ldots$ \\
\hline $250.7 \ldots \ldots \ldots$ & $12.99 \pm 0.52$ & $5.68 \pm 0.23$ & $4.59 \pm 0.50$ \\
\hline $253.6 \ldots \ldots \ldots$ & $11.67 \pm 0.47$ & $5.22 \pm 0.21$ & $3.53 \pm 0.39$ \\
\hline $253.7 \ldots \ldots \ldots$ & $12.52 \pm 0.25$ & $4.94 \pm 0.10$ & $\ldots$ \\
\hline $257.7 \ldots \ldots \ldots$ & $12.09 \pm 0.48$ & $4.47 \pm 0.18$ & $3.45 \pm 0.38$ \\
\hline $259.6 \ldots \ldots \ldots$ & $11.92 \pm 0.48$ & $4.57 \pm 0.18$ & $3.35 \pm 0.37$ \\
\hline $262.7 \ldots \ldots \ldots$ & $12.41 \pm 0.25$ & $4.17 \pm 0.08$ & $\ldots$ \\
\hline $281.6 \ldots \ldots \ldots$ & $13.64 \pm 0.55$ & $5.25 \pm 0.21$ & $4.17 \pm 0.46$ \\
\hline $284.7 \ldots \ldots \ldots$ & $14.68 \pm 0.59$ & $5.89 \pm 0.24$ & $\ldots$ \\
\hline $288.6 \ldots \ldots \ldots$ & $13.25 \pm 0.53$ & $5.45 \pm 0.22$ & $4.74 \pm 0.52$ \\
\hline $429.0 \ldots \ldots \ldots$ & $12.98 \pm 0.52$ & $5.84 \pm 0.23$ & $4.46 \pm 0.49$ \\
\hline $435.0 \ldots \ldots \ldots$ & $13.53 \pm 0.54$ & $5.46 \pm 0.22$ & $4.71 \pm 0.52$ \\
\hline $451.0 \ldots \ldots \ldots$ & $12.84 \pm 0.51$ & $5.51 \pm 0.22$ & $4.05 \pm 0.45$ \\
\hline $458.0 \ldots \ldots \ldots$ & $13.25 \pm 0.53$ & $5.38 \pm 0.22$ & $4.34 \pm 0.48$ \\
\hline $465.0 \ldots \ldots \ldots$ & $12.30 \pm 0.49$ & $4.74 \pm 0.19$ & $4.56 \pm 0.50$ \\
\hline $479.9 \ldots \ldots \ldots$ & $12.81 \pm 0.26$ & $5.04 \pm 0.10$ & $\ldots$ \\
\hline $480.0 \ldots \ldots \ldots$ & $12.68 \pm 0.51$ & $5.10 \pm 0.20$ & $4.67 \pm 0.51$ \\
\hline $483.1 \ldots \ldots \ldots$ & $11.95 \pm 0.48$ & $4.69 \pm 0.19$ & $3.08 \pm 0.34$ \\
\hline $485.9 \ldots \ldots \ldots$ & $12.41 \pm 0.50$ & $4.70 \pm 0.19$ & $3.22 \pm 0.35$ \\
\hline $489.0 \ldots \ldots \ldots$ & $14.03 \pm 0.56$ & $4.92 \pm 0.20$ & $3.99 \pm 0.44$ \\
\hline $493.9 \ldots \ldots \ldots$ & $12.67 \pm 0.25$ & $4.99 \pm 0.10$ & $\ldots$ \\
\hline $494.0 \ldots \ldots \ldots$ & $13.53 \pm 0.54$ & $4.83 \pm 0.19$ & $3.30 \pm 0.36$ \\
\hline $506.8 \ldots \ldots \ldots$ & $11.46 \pm 0.23$ & $4.69 \pm 0.09$ & $\ldots$ \\
\hline
\end{tabular}


TABLE 2-Continued

\begin{tabular}{|c|c|c|c|}
\hline $\begin{array}{c}\text { Julian Date } \\
(-2,450,000) \\
(1)\end{array}$ & $\begin{array}{c}F_{\lambda}(5100 \AA) \\
\left(10^{-15} \operatorname{ergs~s}^{-1} \mathrm{~cm}^{-2} \AA^{-1}\right) \\
(2)\end{array}$ & $\begin{array}{c}F(\mathrm{H} \beta) \\
\left(10^{-13} \mathrm{ergs} \mathrm{s}^{-1} \mathrm{~cm}^{-2}\right) \\
(3)\end{array}$ & $\begin{array}{c}F(\mathrm{He} \mathrm{II} \lambda 4686) \\
\left(10^{-13} \mathrm{ergs} \mathrm{s}^{-1} \mathrm{~cm}^{-2}\right) \\
(4)\end{array}$ \\
\hline $510.0 \ldots \ldots \ldots$ & $12.30 \pm 0.49$ & $4.37 \pm 0.17$ & $3.27 \pm 0.36$ \\
\hline $519.8 \ldots \ldots \ldots$ & $12.51 \pm 0.50$ & $4.74 \pm 0.19$ & $3.68 \pm 0.41$ \\
\hline $520.8 \ldots \ldots \ldots$ & $12.50 \pm 0.25$ & $5.12 \pm 0.10$ & $\ldots$ \\
\hline $521.8 \ldots \ldots \ldots$ & $12.65 \pm 0.51$ & $4.68 \pm 0.19$ & $3.49 \pm 0.38$ \\
\hline $527.8 \ldots \ldots \ldots$ & $10.92 \pm 0.22$ & $4.57 \pm 0.09$ & $\ldots$ \\
\hline $547.6 \ldots \ldots \ldots$ & $11.80 \pm 0.47$ & $4.52 \pm 0.18$ & $3.57 \pm 0.39$ \\
\hline $550.8 \ldots \ldots \ldots$ & $11.74 \pm 0.47$ & $4.47 \pm 0.18$ & $2.96 \pm 0.32$ \\
\hline $551.8 \ldots \ldots \ldots$ & $11.59 \pm 0.46$ & $4.42 \pm 0.18$ & $3.11 \pm 0.34$ \\
\hline $567.6 \ldots \ldots \ldots$ & $10.88 \pm 0.44$ & $4.48 \pm 0.18$ & $3.76 \pm 0.41$ \\
\hline $569.6 \ldots \ldots \ldots$ & $11.76 \pm 0.47$ & $4.31 \pm 0.17$ & $3.12 \pm 0.34$ \\
\hline $576.6 \ldots \ldots \ldots$ & $12.17 \pm 0.49$ & $4.12 \pm 0.17$ & $2.91 \pm 0.32$ \\
\hline $578.6 \ldots \ldots \ldots$ & $12.02 \pm 0.48$ & $4.32 \pm 0.17$ & $3.24 \pm 0.36$ \\
\hline $580.7 \ldots \ldots \ldots$ & $11.97 \pm 0.48$ & $4.91 \pm 0.20$ & $3.28 \pm 0.36$ \\
\hline $582.6 \ldots \ldots \ldots$ & $12.10 \pm 0.48$ & $4.49 \pm 0.18$ & $3.35 \pm 0.37$ \\
\hline $597.7 \ldots \ldots \ldots$ & $12.57 \pm 0.50$ & $4.24 \pm 0.17$ & $2.92 \pm 0.32$ \\
\hline $600.6 \ldots \ldots \ldots$ & $12.44 \pm 0.50$ & $4.15 \pm 0.17$ & $2.59 \pm 0.28$ \\
\hline $602.6 \ldots \ldots \ldots$ & $11.62 \pm 0.47$ & $4.61 \pm 0.18$ & $3.53 \pm 0.39$ \\
\hline $604.6 \ldots \ldots \ldots$ & $12.00 \pm 0.48$ & $4.50 \pm 0.18$ & $3.59 \pm 0.40$ \\
\hline $608.6 \ldots \ldots \ldots$ & $11.96 \pm 0.48$ & $4.16 \pm 0.17$ & $3.39 \pm 0.37$ \\
\hline $610.6 \ldots \ldots \ldots$ & $10.99 \pm 0.44$ & $4.24 \pm 0.17$ & $2.80 \pm 0.31$ \\
\hline $627.6 \ldots \ldots \ldots$ & $11.26 \pm 0.45$ & $4.01 \pm 0.16$ & $2.92 \pm 0.32$ \\
\hline $630.7 \ldots \ldots \ldots$ & $11.14 \pm 0.45$ & $3.88 \pm 0.16$ & $2.49 \pm 0.27$ \\
\hline $635.6 \ldots \ldots \ldots$ & $11.87 \pm 0.47$ & $4.24 \pm 0.17$ & $2.37 \pm 0.26$ \\
\hline $641.6 \ldots \ldots \ldots$ & $12.33 \pm 0.49$ & $4.71 \pm 0.19$ & $3.20 \pm 0.35$ \\
\hline $643.6 \ldots \ldots \ldots$ & $10.75 \pm 0.43$ & $4.46 \pm 0.18$ & $2.83 \pm 0.31$ \\
\hline $775.0 \ldots \ldots \ldots$ & $13.29 \pm 0.27$ & $5.04 \pm 0.10$ & $\ldots$ \\
\hline $777.0 \ldots \ldots \ldots$ & $12.69 \pm 0.51$ & $5.18 \pm 0.21$ & $3.61 \pm 0.40$ \\
\hline $782.0 \ldots \ldots \ldots$ & $12.86 \pm 0.51$ & $5.41 \pm 0.22$ & $3.83 \pm 0.42$ \\
\hline $787.0 \ldots \ldots \ldots$ & $13.49 \pm 0.54$ & $5.27 \pm 0.21$ & $4.20 \pm 0.46$ \\
\hline $810.1 \ldots \ldots \ldots$ & $14.06 \pm 0.56$ & $3.95 \pm 0.16$ & $2.97 \pm 0.33$ \\
\hline $834.0 \ldots \ldots \ldots$ & $12.00 \pm 0.48$ & $4.85 \pm 0.19$ & $2.87 \pm 0.31$ \\
\hline $838.1 \ldots \ldots \ldots$ & $11.44 \pm 0.46$ & $4.59 \pm 0.18$ & $2.99 \pm 0.33$ \\
\hline $839.0 \ldots \ldots \ldots$ & $11.89 \pm 0.24$ & $4.89 \pm 0.10$ & $\ldots$ \\
\hline $840.1 \ldots \ldots \ldots$ & $12.50 \pm 0.50$ & $4.91 \pm 0.20$ & $3.40 \pm 0.37$ \\
\hline $843.0 \ldots \ldots \ldots$ & $12.45 \pm 0.50$ & $4.87 \pm 0.19$ & $3.29 \pm 0.36$ \\
\hline $847.0 \ldots \ldots \ldots$ & $12.33 \pm 0.49$ & $4.82 \pm 0.19$ & $3.20 \pm 0.35$ \\
\hline $867.0 \ldots \ldots \ldots$ & $12.25 \pm 0.49$ & $4.20 \pm 0.17$ & $2.86 \pm 0.31$ \\
\hline $869.0 \ldots \ldots \ldots$ & $12.55 \pm 0.50$ & $3.85 \pm 0.15$ & $2.53 \pm 0.28$ \\
\hline $873.0 \ldots \ldots \ldots$ & $12.15 \pm 0.49$ & $4.09 \pm 0.16$ & $3.01 \pm 0.33$ \\
\hline $875.8 \ldots \ldots \ldots$ & $12.29 \pm 0.25$ & $4.49 \pm 0.09$ & $\ldots$ \\
\hline $876.0 \ldots \ldots \ldots$ & $12.58 \pm 0.50$ & $4.31 \pm 0.17$ & $3.09 \pm 0.34$ \\
\hline $877.0 \ldots \ldots .$. & $12.44 \pm 0.50$ & $4.37 \pm 0.17$ & $3.09 \pm 0.34$ \\
\hline $885.8 \ldots \ldots \ldots$ & $11.54 \pm 0.23$ & $4.18 \pm 0.08$ & $\ldots$ \\
\hline $892.8 \ldots \ldots \ldots$ & $12.14 \pm 0.49$ & $4.54 \pm 0.18$ & $3.05 \pm 0.34$ \\
\hline $906.9 \ldots \ldots \ldots$ & $11.44 \pm 0.46$ & $4.53 \pm 0.18$ & $2.60 \pm 0.28$ \\
\hline $921.9 \ldots \ldots \ldots$ & $11.61 \pm 0.46$ & $4.53 \pm 0.18$ & $2.93 \pm 0.32$ \\
\hline $926.8 \ldots \ldots \ldots$ & $11.13 \pm 0.22$ & $4.43 \pm 0.09$ & $\ldots$ \\
\hline $930.8 \ldots \ldots \ldots$ & $11.43 \pm 0.46$ & $4.01 \pm 0.16$ & $2.74 \pm 0.30$ \\
\hline $935.9 \ldots \ldots \ldots$ & $11.92 \pm 0.48$ & $4.51 \pm 0.18$ & $\ldots$ \\
\hline $936.6 \ldots \ldots \ldots$ & $11.23 \pm 0.45$ & $4.39 \pm 0.18$ & $2.65 \pm 0.29$ \\
\hline $949.6 \ldots \ldots \ldots$ & $12.03 \pm 0.48$ & $5.04 \pm 0.20$ & $3.41 \pm 0.38$ \\
\hline $961.6 \ldots \ldots \ldots$ & $11.32 \pm 0.45$ & $4.90 \pm 0.20$ & $3.09 \pm 0.34$ \\
\hline $966.7 \ldots \ldots \ldots$ & $11.60 \pm 0.46$ & $4.39 \pm 0.18$ & $3.00 \pm 0.33$ \\
\hline $980.7 \ldots \ldots \ldots$ & $11.72 \pm 0.21$ & $4.73 \pm 0.08$ & $3.13 \pm 0.34$ \\
\hline $983.7 \ldots \ldots \ldots$ & $11.41 \pm 0.46$ & $4.46 \pm 0.18$ & $2.60 \pm 0.29$ \\
\hline $988.6 \ldots \ldots \ldots$ & $11.56 \pm 0.46$ & $4.30 \pm 0.17$ & $2.78 \pm 0.31$ \\
\hline $991.7 \ldots \ldots \ldots$ & $11.35 \pm 0.45$ & $4.15 \pm 0.17$ & $3.08 \pm 0.34$ \\
\hline $994.6 \ldots \ldots \ldots$ & $11.37 \pm 0.46$ & $4.11 \pm 0.16$ & $2.56 \pm 0.28$ \\
\hline $1009.6 \ldots \ldots$ & $10.98 \pm 0.44$ & $3.76 \pm 0.15$ & $2.50 \pm 0.28$ \\
\hline $1012.6 \ldots \ldots$ & $12.18 \pm 0.49$ & $3.70 \pm 0.15$ & $2.98 \pm 0.33$ \\
\hline $1019.6 \ldots \ldots$ & $12.75 \pm 0.51$ & $4.20 \pm 0.17$ & $3.33 \pm 0.37$ \\
\hline $1022.6 \ldots \ldots$ & $12.09 \pm 0.48$ & $4.57 \pm 0.18$ & $3.02 \pm 0.33$ \\
\hline
\end{tabular}


component of $\mathrm{H} \beta$, which is of course expected to be constant.

We have also measured the flux in the He II $\lambda 4686$ line. Only set B spectra are suitable for this measurement, since set A spectra do not extend shortward far enough to provide a suitable short-wavelength continuum point. The He II flux was measured by adopting a linear underlying continuum between $\sim 4447$ and $\sim 4775 \AA$ and integrating the flux above this continuum between 4613 and $4772 \AA$.

We then compare the independent light curves from the two sets of data to identify small systematic flux differences between the sets, as we have done in many previous experiments (see Peterson et al. 1999 and references therein). We attribute these small relative flux offsets to aperture effects, although the procedure we use also corrects for other unidentified systematic differences between data sets. We define a point-source correction factor $\varphi$ by the equation

$$
F(\mathrm{H} \beta)_{\mathrm{true}}=\varphi F(\mathrm{H} \beta)_{\mathrm{observed}} .
$$

This factor accounts for the fact that different apertures result in different amounts of light loss for the point-spread function (which describes the surface brightness distribution of both the broad lines and the AGN continuum source) and the partially extended narrow-line region (NLR). After correcting for aperture effects on the point-spread functionto-narrow-line ratio, another correction needs to be applied to adjust for the different amounts of starlight admitted by different apertures. An extended source correction $G$ is thus defined as

$$
F_{\lambda}(5100)_{\text {true }}=\varphi F_{\lambda}(5100)_{\text {observed }}-G .
$$

The value of $G$ is essentially the nominal difference in the contaminating host galaxy flux between the two spectrograph entrance apertures employed.

This intercalibration procedure is accomplished by comparing pairs of nearly simultaneous observations from the two data sets to determine $\varphi$ and $G$. In practice, the interval that we define as "nearly simultaneous" is 2 days or less, which means that, in principle, any real variability that occurs on timescales this short tends to be somewhat suppressed by the process that allows us to merge the two data sets. In this case, the adjustment has very little impact on the final results because nearly all of the pairs of data separated by 2 days or less are from data set B and thus have not been adjusted relative to one another. We find that the best-fit constants for set $\mathrm{B}$ relative to set $\mathrm{A}$ are $\varphi=0.982 \pm 0.048$ and $G=(-1.243 \pm 0.729) \times 10^{-15} \mathrm{ergs}$ $\mathrm{s}^{-1} \mathrm{~cm}^{-2} \AA^{-1}$. Both the $\mathrm{H} \beta$ and He II $\lambda 4686$ fluxes are adjusted as in equation (5); the fact that the factor $\varphi$ is so close to unity indicates that most of the NLR in this galaxy arises within a few arcseconds of the nucleus.

The final continuum $F_{\lambda}(5100 \AA)$ and $\mathrm{H} \beta$ emission-line fluxes are given in Table 2. Simultaneous (to within 0.1 day) measurements were averaged, weighted by the reciprocal of their variances.

\section{ANALYSIS}

\subsection{Continuum Variability}

The light curves listed in Table 2 are plotted in Figure 1, along with contemporaneous $2-10 \mathrm{keV}$ X-ray light curves. The data shown here span three observing seasons, beginning in 1996 January and ending in 1998 July. A summary of the general variability characteristics is given in Table 3.
For the complete data base and for individual subsets of the data as given in column (1), columns (2)-(4) give, respectively, the number of individual observations and the average and median time intervals between them. The mean flux is given in column (5), and columns (6) and (7) give two widely used measures of the variability: $F_{\text {var }}$, the rms fractional variability corrected for measurement error, as defined by Rodríguez-Pascual et al. (1997), and $R_{\max }$, the ratio of maximum to minimum flux, respectively. Both the $F_{\text {var }}$ and $R_{\max }$ parameters are affected by contamination of the measured quantities by constant-flux components; the optical continuum values are somewhat diluted by the constant contribution of the underlying host galaxy, and the emission-line values are affected by both narrow-line contributions and probably slowly varying Fe II emission as well. In any case, these will have only a modest effect on $F_{\text {var }}$ and $R_{\max }$, and inspection of Table 3 shows clearly that the largeamplitude, rapid variations that characterize X-rays in NLS1s are much less pronounced in the optical spectrum.

While there is a clear lack of correlated short timescale behavior of the X-ray and optical continua, the light curves in Figure 1 suggest that a correlation on longer timescales is possible. To test this quantitatively, we have suppressed the rapid variations by smoothing both the optical continuum and X-ray light curves with a rectangular function of width 30 days (as shown in Fig. 2), similar to what was done by Maoz, Edelson, \& Nandra (2000) in a comparison of X-ray and optical variability in the Seyfert 1 galaxy NGC 3516. Cross-correlation of the overlapping parts of these light curves using the methodology described in the next section yields a lag of the optical variations relative to the X-ray of $\tau=6_{-112}^{+62}$ days with a correlation coefficient $r_{\max }=0.74$, i.e., the mean X-ray and optical fluxes are indeed correlated once the high-frequency variability is suppressed. The lag between variations in the two wave bands is highly uncertain but consistent with zero or any small time lag expected in the continuum emitting region.

\subsection{Emission-Line Variability}

Comparison of the optical continuum and emission-line light curves shows that the variations in each are quite similar, indicating that the time delay between them is small. The time delay between continuum and emission-line

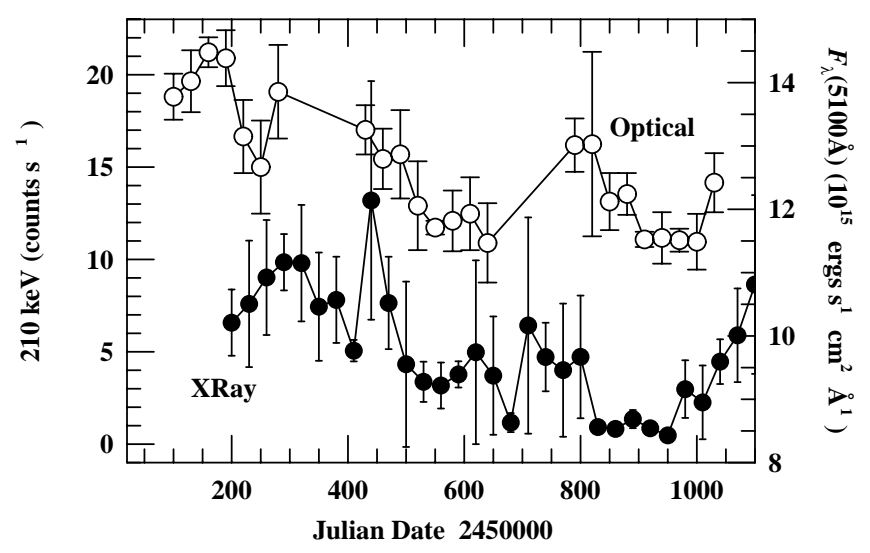

FIG. 2.-Mean X-ray ( filled circles, flux scale on left axis) and optical continuum (open circles, flux scale on right axis) light curves, as in Fig. 1, smoothed with a rectangular function of width 30 days. This shows that when the rapid X-ray variability is suppressed by averaging, the X-ray and optical variations are correlated on timescales of several weeks and longer. 
TABLE 3

\begin{tabular}{|c|c|c|c|c|c|c|}
\hline \multicolumn{7}{|c|}{ VARIABILITY PARAMETERS } \\
\hline $\begin{array}{l}\text { Subset } \\
\text { (1) }\end{array}$ & $\begin{array}{l}\text { Number of Epochs } \\
\text { (2) }\end{array}$ & $\begin{array}{c}\Delta t_{\text {ave }} \\
\text { (days) } \\
\text { (3) }\end{array}$ & $\begin{array}{c}\Delta t_{\text {med }} \\
\text { (days) } \\
\text { (4) }\end{array}$ & $\begin{array}{l}\text { Mean Flux } \\
\text { (5) }\end{array}$ & $\begin{array}{l}F_{\mathrm{var}} \\
(6)\end{array}$ & $\begin{array}{l}R_{\max } \\
\text { (7) }\end{array}$ \\
\hline \multicolumn{7}{|c|}{ X-Ray $(2-10 \mathrm{keV})$} \\
\hline All ............ & 140 & 7.5 & 7.3 & $6.20 \pm 3.85$ & 0.618 & $42.85 \pm 16.36$ \\
\hline Year $1 \ldots \ldots \ldots$ & 33 & 2.8 & 0.7 & $7.87 \pm 3.25$ & 0.411 & $5.766 \pm 0.459$ \\
\hline Year $2 \ldots \ldots \ldots$ & 20 & 11.5 & 13.0 & $6.42 \pm 4.95$ & 0.770 & $17.68 \pm 2.03$ \\
\hline Year $3 \ldots \ldots$. & 19 & 14.1 & 14.1 & $1.97 \pm 1.81$ & 0.906 & $14.79 \pm 5.65$ \\
\hline \multicolumn{7}{|c|}{ Continuum $(5100 \AA)$} \\
\hline All ............ & 126 & 7.4 & 3.1 & $12.74 \pm 1.08$ & 0.077 & $1.397 \pm 0.079$ \\
\hline Year $1 \ldots \ldots .$. & 51 & 3.9 & 3.0 & $13.66 \pm 0.82$ & 0.050 & $1.287 \pm 0.073$ \\
\hline Year $2 \ldots \ldots \ldots$ & 38 & 5.8 & 3.2 & $12.16 \pm 0.76$ & 0.050 & $1.305 \pm 0.074$ \\
\hline Year $3 \ldots \ldots \ldots$ & 37 & 6.9 & 4.5 & $12.06 \pm 0.69$ & 0.043 & $1.281 \pm 0.072$ \\
\hline Subset $1 \ldots \ldots$ & 29 & 2.8 & 2.2 & $13.38 \pm 0.92$ & 0.059 & $1.287 \pm 0.073$ \\
\hline \multicolumn{7}{|c|}{$\mathrm{H} \beta$} \\
\hline All ............ & 126 & 7.4 & 3.1 & $4.77 \pm 0.48$ & 0.095 & $1.592 \pm 0.092$ \\
\hline Year $1 \ldots . . .$. & 51 & 3.9 & 3.0 & $4.91 \pm 0.48$ & 0.092 & $1.423 \pm 0.064$ \\
\hline Year $2 \ldots \ldots \ldots$ & 38 & 5.8 & 3.2 & $4.65 \pm 0.44$ & 0.086 & $1.505 \pm 0.086$ \\
\hline Year $3 \ldots \ldots .$. & 37 & 6.9 & 4.5 & $4.50 \pm 0.42$ & 0.086 & $1.462 \pm 0.084$ \\
\hline Subset $1 \ldots \ldots$ & 29 & 2.8 & 2.2 & $5.02 \pm 0.51$ & 0.096 & $1.389 \pm 0.062$ \\
\hline \multicolumn{7}{|c|}{ He II $\lambda 4686$} \\
\hline All .............. & 93 & 10.1 & 4.5 & $3.50 \pm 0.67$ & 0.154 & $2.038 \pm 0.316$ \\
\hline Year $1 \ldots . . .$. & 29 & 6.9 & 4.0 & $4.08 \pm 0.52$ & 0.064 & $1.660 \pm 0.258$ \\
\hline Year $2 \ldots \ldots \ldots$ & 33 & 6.7 & 3.6 & $3.42 \pm 0.62$ & 0.143 & $1.987 \pm 0.309$ \\
\hline Year $3 \ldots . . .$. & 31 & 8.2 & 5.0 & $3.04 \pm 0.38$ & 0.057 & $1.680 \pm 0.263$ \\
\hline Subset $1 \ldots \ldots$ & 17 & 4.8 & 3.5 & $4.08 \pm 0.65$ & 0.114 & $1.660 \pm 0.258$ \\
\hline
\end{tabular}

${ }^{\text {a }} \mathrm{X}$-ray fluxes in counts $\mathrm{s}^{-1}$ in the $2-10 \mathrm{keV}$ band. Other fluxes are in units as in Table 2.

variations can be quantified by cross-correlation of the light curves. During the first year, there is a period from JD $2,450,183$ to JD 2,450,262 in which the light curves are well sampled and the character of the variations permits an accurate cross-correlation measurement. In Table 3, we refer to this subset of data as "subset 1," and we plot this part of the light curves in an expanded form in Figure 3. We cross-correlate the data shown here by using the interpolation cross-correlation function (ICCF) method of Gaskell \& Sparke (1986) and Gaskell \& Peterson (1987) and the discrete correlation function (DCF) method of Edelson \& Krolik (1988), where in both cases we employ the specific implementation described by White \& Peterson (1994).

The results of the cross-correlation analysis are summarized in Table 4, and the cross-correlation functions are shown in Figure 4. For both $\mathrm{H} \beta$ and $\mathrm{He}$ II $\lambda 4686$, Table 4 gives the ICCF centroid $\tau_{\text {cent }}$ and the location $\tau_{\text {peak }}$ of the maximum value of the correlation coefficient $r_{\max }$. The cen-

TABLE 4

Cross-Correlation Results aNd Virial Mass Estimates

\begin{tabular}{ccc}
\hline \hline $\begin{array}{c}\text { Parameter } \\
(1)\end{array}$ & $F(\mathrm{H} \beta)$ & $F(\mathrm{He}$ II $\lambda 4686)$ \\
$(2)$ & $(3)$ \\
\hline$\tau_{\text {cent }}($ days $) \ldots \ldots \ldots \ldots$. & $5.92_{-1.96}^{+3.13}$ & $4.49_{-5.60}^{+4.91}$ \\
$\tau_{\text {peak }}($ days $) \ldots \ldots \ldots \ldots$ & $4.6_{-1.5}^{+4.5}$ & $5.9_{-4.8}^{+8.9}$ \\
$r_{\text {max }} \ldots \ldots \ldots \ldots \ldots \ldots$. & 0.800 & 0.767 \\
$V_{\text {FWHM }}\left(\mathrm{km} \mathrm{s}^{-1}\right) \ldots \ldots$. & $1110 \pm 190$ & $5430 \pm 510$ \\
$M_{\text {vir }}\left(10^{6} M_{\odot}\right) \ldots \ldots$. & $1.1_{-0.5}^{+0.8}$ & $19.4_{-24.4}^{+21.5}$ \\
\hline
\end{tabular}

troid $\tau_{\text {cent }}$ is computed using all points near $\tau_{\text {peak }}$ with values greater than $0.8 r_{\max }$. The uncertainties quoted for $\tau_{\text {cent }}$ and $\tau_{\text {peak }}$ are based on the model-independent Monte Carlo method described by Peterson et al. (1998).

By combining this lag with the Doppler width of the emission line, we can estimate a virial mass, as in equation (4); for consistency with Wandel et al. (1999) and Kaspi et al. (2000), we use $f=3 / \sqrt{2}$ in equation (4). Since the broad emission-line features are comprised of a number of different components (or contaminants), it is desirable to measure the Doppler width of only the variable part of the emission line. In order to isolate the variable part of the emission line and exclude constant components (such as contamination from the NLR), we measure the relevant line widths in the rms spectrum constructed from all of the set B spectra in subset 1 . The mean spectrum is constructed by averaging all $N(=18)$ set B spectra in subset 1, i.e.,

$$
\bar{F}(\lambda)=\frac{1}{N} \sum_{i=1}^{N} F_{i}(\lambda),
$$

where $F_{i}(\lambda)$ is the flux density (in ergs $\mathrm{s}^{-1} \mathrm{~cm}^{-2} \AA^{-1}$ ) at wavelength $\lambda$ in the $i$ th spectrum. The rms spectrum is similarly constructed as

$$
\sigma(\lambda)=\left\{\left(\frac{1}{N-1}\right) \sum_{i=1}^{N}\left[F_{i}(\lambda)-\bar{F}(\lambda)\right]^{2}\right\}^{1 / 2} .
$$

The mean and rms spectra are shown as the top two panels in Figure 5. The widths of the $\mathrm{H} \beta$ and $\mathrm{He}$ II $\lambda 4686$ emission lines $\left(V_{\mathrm{FWHM}}\right)$ are given in Table 4 as Doppler widths in the 


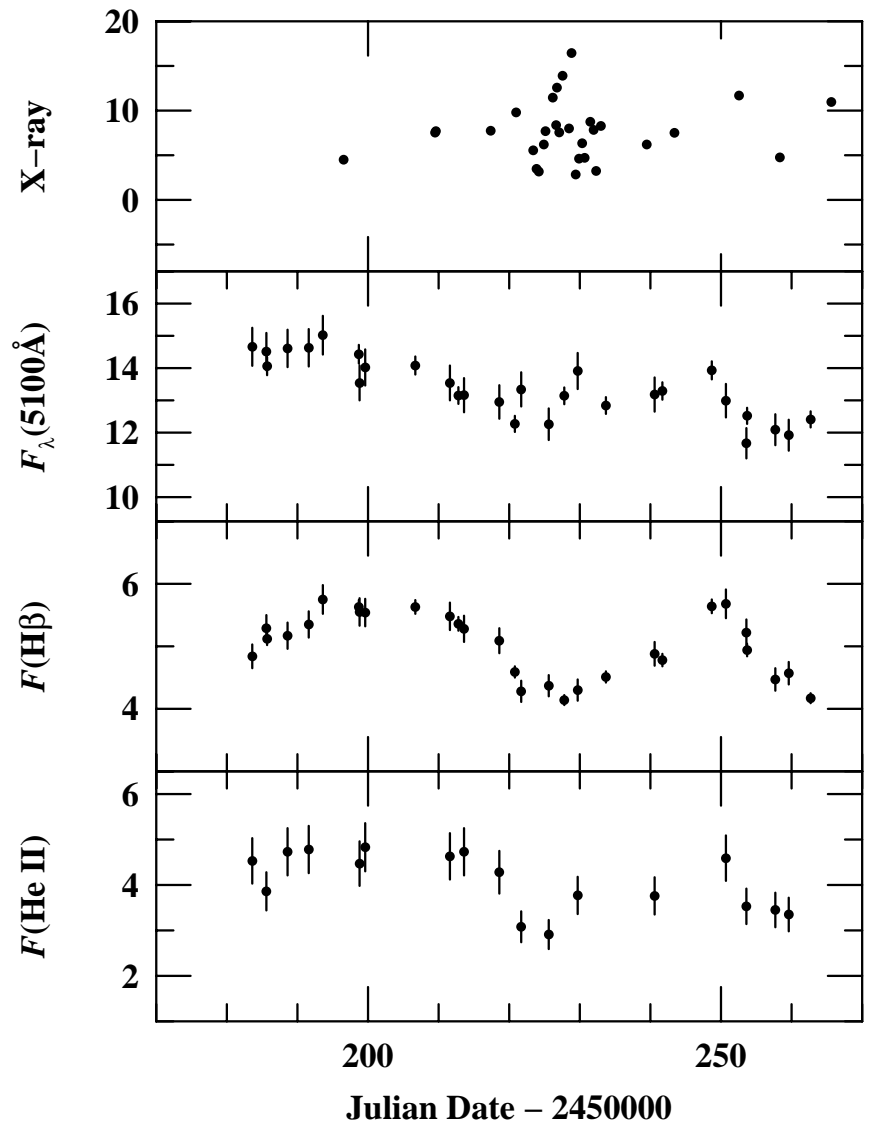

FIG. 3.-Light curves in Fig. 1 are expanded to show the well-sampled region used in the optical continuum/emission-line cross-correlation analysis (subset 1) between JD 2,450,183 and JD 2,450,262. Units are as in Fig. 1.

rest frame of NGC 4051. A virial mass is then computed as described by Wandel et al. (1999). On the basis of the $\mathrm{H} \beta$ variations, a mass of $1.1_{-0.5}^{+0.8} \times 10^{6} M_{\odot}$ is inferred; unfortunately, however, the extremely large uncertainty in the He II $\lambda 4686$ lag renders the virial mass obtained from it not very enlightening, but it is consistent with the $\mathrm{H} \beta$ result.

It is also important to keep in mind that because of the unknown geometry and kinematics of the BLR, the virial mass is reliable to only about an order of magnitude, i.e., the systematic uncertainties are much larger than the errors quoted here. However, an independent estimate of the inclination of the system has been made by Christopoulou et al. (1997) on the basis of the NLR morphology and kinematics. These authors model the NLR as an outflowing biconical region of inclination $50^{\circ}$ and half-opening angle $23^{\circ}$. If the BLR and NLR axes are co-aligned, then correcting the virial mass for inclination gives a central mass of $1.4_{-0.6}^{+1.0}$ $\times 10^{6} M_{\odot}$.

There are a number of important features in the rms spectrum that deserve attention:

1. The constant components, such as the [O III] $\lambda \lambda 4959$, 5007 narrow lines that are so prominent in the mean spectrum, are absent, as expected, in the rms spectrum (except for weak residuals that reflect the accuracy to which accurate flux calibration can be achieved).

2. The weak broad wings of $\mathrm{H} \beta$ that can be seen in the mean spectrum are much weaker in the rms spectrum, i.e., the line core is more variable than the line wings. This could

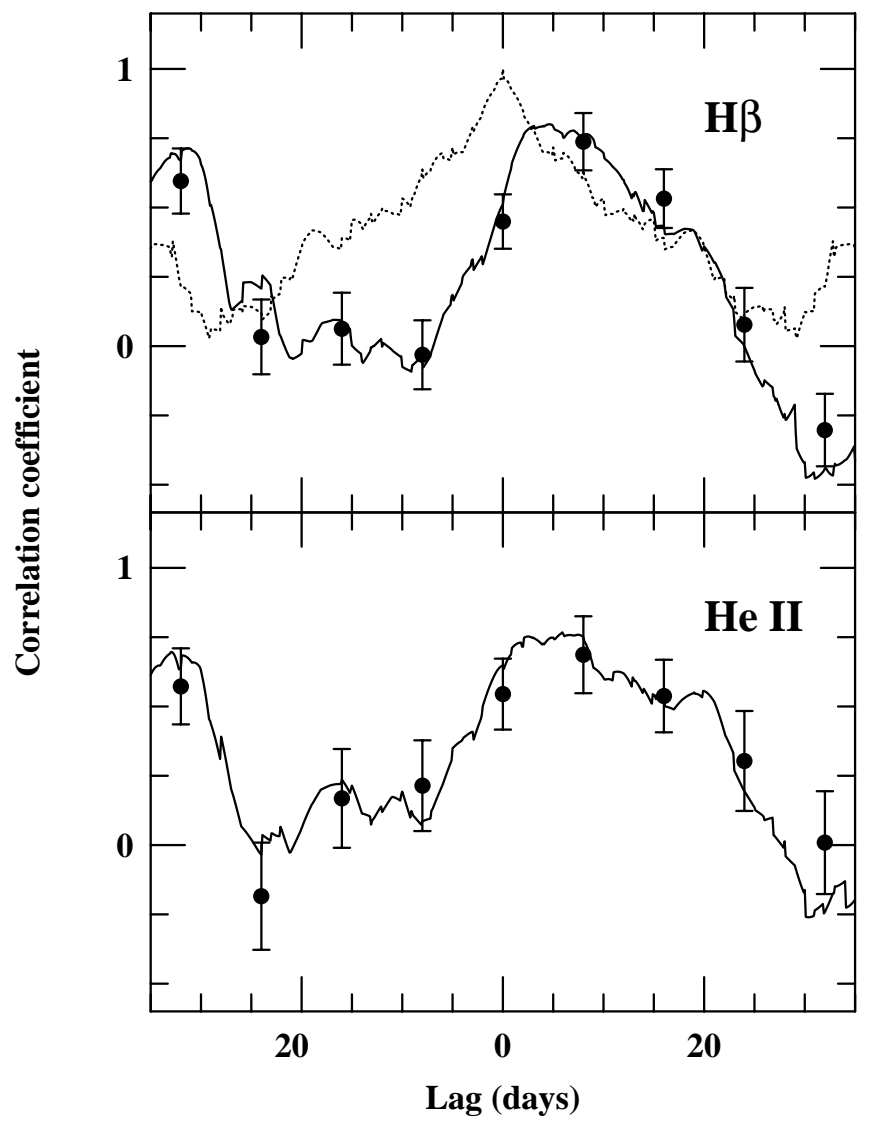

Fig. 4.-Emission-line cross-correlation functions are shown, interpolation cross-correlation function as solid lines and discrete correlation function values as points with associated error bars. The top panel shows the $5100 \AA$ continuum $\mathrm{H} \beta$ cross-correlation, along with the continuum autocorrelation function shown as a dotted line. The bottom panel shows the $5100 \AA$ continuum He II $\lambda 4686$ cross-correlation. Peak and centroid values are given in Table 4.

occur if the higher velocity material is much farther away from the ionizing source, or if some significant component of the high-velocity gas is optically thin (e.g., Shields, Ferland, \& Peterson 1995); on physical grounds, we prefer the latter explanation.

3. The rms spectrum also shows that the optical Fe II emission varied little, if at all, during this period. The optical spectra of AGNs have broad blended Fe II features that extend from just longward of $\mathrm{H} \gamma$ to just shortward of $\mathrm{H} \beta$ and over the range $\sim 5100-5600 \AA$. In the mean spectrum, these $\mathrm{Fe}$ II emission features are quite strong, which makes it hard to isolate the He II $\lambda 4686$ emission. The Fe II blends are, however, virtually absent in the rms spectrum.

4. He II $\lambda 4686$ is very prominent in the rms spectrum and is much broader than $\mathrm{H} \beta$. The rest-frame width of this line in the rms spectrum is $\sim 5430 \mathrm{~km} \mathrm{~s}^{-1}$, very typical of the line widths seen in normal Seyfert 1 galaxies. The centroid of the He II $\lambda 4686$ is strongly blueshifted relative to $\mathrm{H} \beta$, by about $1400 \mathrm{~km} \mathrm{~s}^{-1}$. In order to demonstrate that these properties of the He II $\lambda 4686$ profile are real, we also computed the mean and rms spectra based on (1) set A spectra obtained at the same time and (2) set B spectra obtained during the 1997 monitoring season (year 2). The rms spectra from these subsets, shown in the bottom two panels of Figure 5, show the same He II $\lambda 4686$ profile characteristics seen in the set B subset 1 data shown in the second panel. 


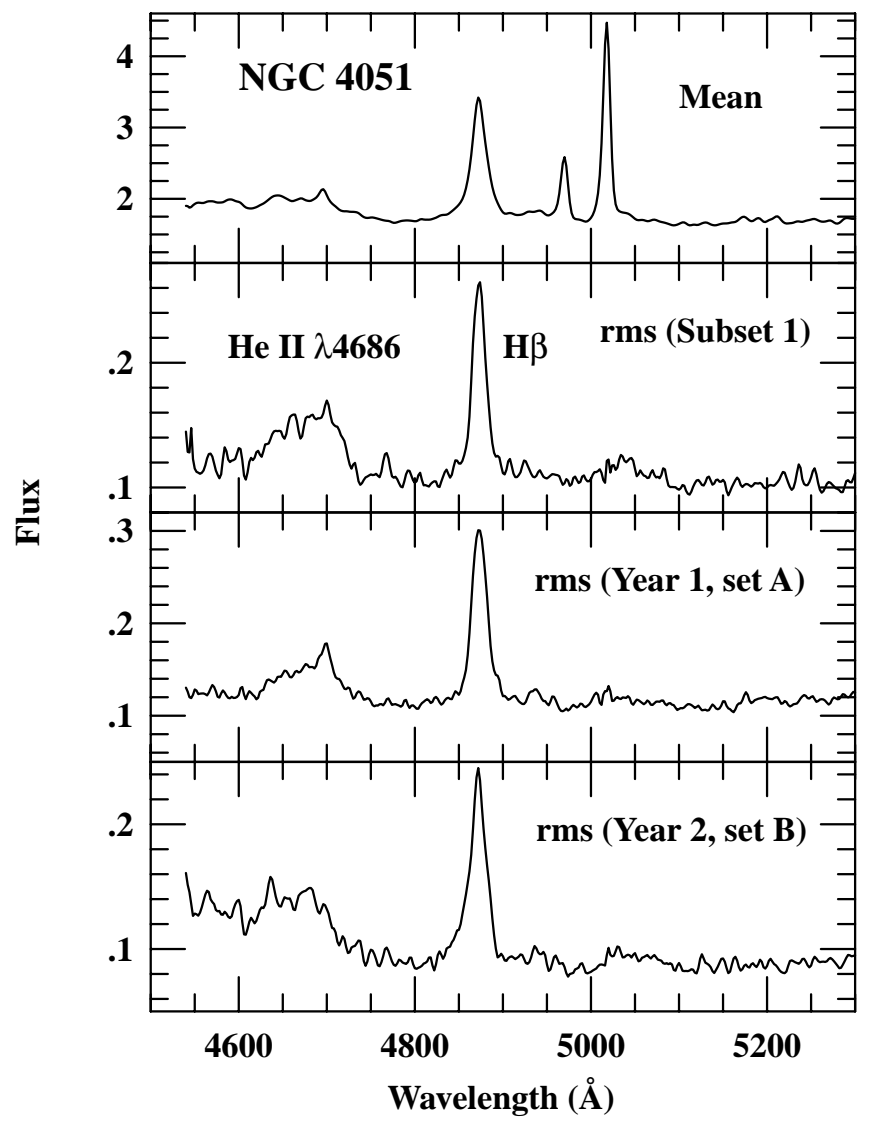

FIG. 5.-Mean (top panel) and rms (second panel) spectra of NGC 4051 based on individual spectra obtained during the well-sampled interval between JD 2,450,183 and JD 2,450,262 (subset 1), as in Fig. 3. The spectra are plotted in the observed frame and in units of $10^{-15} \mathrm{ergs} \mathrm{s}^{-1} \mathrm{~cm}^{-2} \AA^{-1}$, as in Table 2 . These are based only on the data from set B. The He II $\lambda 4686$ line is very broad $\left(\mathrm{FWHM} \approx 5430 \mathrm{~km} \mathrm{~s}^{-1}\right.$ ) and blueshifted (by $\sim 1400 \mathrm{~km}$ $\mathrm{s}^{-1}$ relative to the narrow emission lines); these characteristics are also seen in the rms spectrum based on contemporaneous spectra from set $\mathrm{A}$ (third panel) and on set B spectra obtained the next year (bottom panel).

\section{DISCUSSION}

\subsection{The Continuum}

As described in the previous section, the X-ray and optical continuum variations are not closely coupled on short timescales. The X-ray continuum shows large-scale variations on short timescales, as is typical of the NLS1 class. The rapid variations seen in the X-ray are not detected in the optical, as has previously been reported by Done et al. (1990) for this same source and by Young et al. (1999) for IRAS 13224-3809. However, if we average over the short-timescale flares, then the X-ray and optical continuum variations do seem to be coupled, though the time resolution of this experiment is insufficient to determine whether there is any lag between them at the level of days or less. The absence of strong coupling of the hard X-ray and optical continuum variations argues against reprocessing models in which hard X-rays are absorbed by a dense plasma (such as the accretion disk) and the energy is reradiated at longer wavelengths (Guilbert \& Rees 1988). The $\mathrm{X}$-ray variations are more suggestive of localized flaring types of activity that may arise in a patchy corona above the accretion disk.
It has already been pointed out based on these same $R X T E$ data (Uttley et al. 1999) that the X-ray continuum of NGC 4051 virtually "turned off" in early 1998 (around JD 2,450,800; see Fig. 1). However, the optical spectroscopic data show that the optical continuum and emission lines (and therefore, by inference, the ionizing UV continuum) did not disappear at the same time. This seems in a sense rather suggestive of the kind of behavior that has been seen in Galactic black hole systems such as GRO J1655-40 (Orosz et al. 1997). A possible interpretation of the behavior of NGC 4051 is that the inner X-ray-producing (though not necessarily by purely thermal emission) part of the accretion disk in NGC 4051 has entered an advection-dominated accretion flow (ADAF) state, in which radiation is emitted with very low efficiency (Narayan \& Yi 1994; Narayan et al. 1998). This implies that there is a transition radius $r_{\text {tran }}$ inside of which the disk is an ADAF and outside of which it radiates efficiently, perhaps like a classical thin disk (Shakura \& Sunyaev 1973). The persistence of the optical continuum and emission lines suggests that this transition radius is somewhere between the regions that are most responsible for the soft $\mathrm{X}$-rays and the $\mathrm{H}$-ionizing continuum. We comment on this further in $\S 4.3$ below.

\subsection{The Virial Mass and Implications for NLS1s}

As noted above, reverberation-based size estimates for the broad emission lines and resulting virial mass estimates provide a potential means of distinguishing among the various NLS1 models. In Figure 6, we show the relationship between the BLR radius as measured from the $\mathrm{H} \beta$ lag as a function of the optical continuum luminosity for all AGNs with Balmer-line lags known to reasonable accuracy. All data used here are taken from the compilation of Kaspi et al. (2000), though their parameters for NGC 4051 are superseded by the values reported here. This compilation contains six additional AGNs that could in principle be classified as NLS1s, as they meet the criterion $V_{\mathrm{FWHM}} \lesssim$ $2000 \mathrm{~km} \mathrm{~s}^{-1}$. These sources, which we shall refer to below as "narrow-line objects," are the Seyfert galaxies Mrk 335

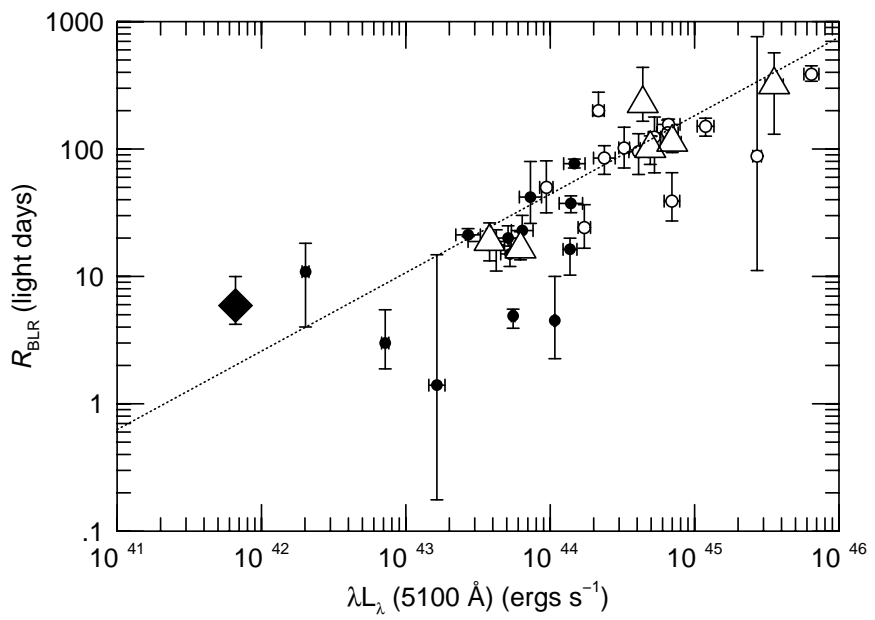

Fig. 6. - Relationship between the size of the broad Balmer-lineemitting region and optical luminosity for AGNs. The filled circles are Seyfert galaxies from Wandel et al. (1999), and the open circles are QSOs from Kaspi et al. (2000). The large triangles are the AGNs from the same sources whose broad lines have widths less than $\sim 2000 \mathrm{~km} \mathrm{~s}^{-1}$, i.e., the Seyfert galaxies Mrk 335 and Mrk 110 and the QSOs PG 0026+120, PG $1211+143$, PG $1351+640$, and PG $1704+608$. NGC 4051 is shown as a filled diamond. 
and Mrk 110 (see Wandel et al. 1999) and the quasi-stellar objects (QSOs) PG $0026+120$, PG $1211+143$, PG $1351+640$, and PG 1704 + 608 (from Kaspi et al. 2000).

The best-fit regression line $\left(R_{\mathrm{BLR}} \propto L^{0.62 \pm 0.02}\right)$, based on all objects from Table 5 of Kaspi et al. (2000) except NGC 4051, is shown as a dotted line. NGC 4051 lies approximately $2.8 \sigma$ above this regression line, although all of the other narrow-line objects clearly fall in the locus defined by the AGNs with broader lines. It is difficult to argue that NGC 4051 is somehow different from the other AGNs, as there are several AGNs that have large displacements from the regression line. This is reflected in the large value of the reduced $\chi^{2}$ statistic, $\chi_{v}^{2}=15.7$, for this fit.

In Figure 7, we plot the mass-luminosity relationship for the AGNs from Kaspi et al. (2000), and we show (1) the best-fit regression line based on all objects except the seven narrow-line objects and (2) that based on the narrow-line objects alone. Formally, the slopes $\alpha$ (for $M \propto L^{\alpha}$ ) are significantly different, with $\alpha=0.46 \pm 0.06$ for the narrow-line objects (including NGC 4051) and $\alpha=0.27 \pm 0.03$ for the others. These two fits are separated by typically an order of magnitude in black hole mass; the black holes in the narrow-line objects are about a factor of 10 lower than those of other AGNs of comparable luminosity.

How well do these results allow us to distinguish among the various explanations for the NLS1 phenomenon? We consider the possibilities:

1. Do the BLRs of NLS1s have anomalously large radii? The position of NGC 4051 in Figure 6 might suggest that this is possible, but the distribution of other narrow-line objects does not support this. Furthermore, as noted above, the scatter in the BLR radius-luminosity relationship is very large, and NGC 4051 is in a statistical sense not the largest outlier in this relationship (simply because other sources have smaller uncertainties in their measured lags).

2. Are NLS1s simply low-inclination systems? If the BLR is a flattened system, at low inclination (i.e., nearly face-on) the line widths will be decreased by a factor of $\sin i$, but the measured emission-line lags will be relatively unaffected. On the other hand, assuming that the UV-optical continuum arises in an accretion disk at the same inclination, the apparent UV-optical luminosity is higher at lower inclination (e.g., see Fig. 32 of Netzer 1990). Thus, relative to

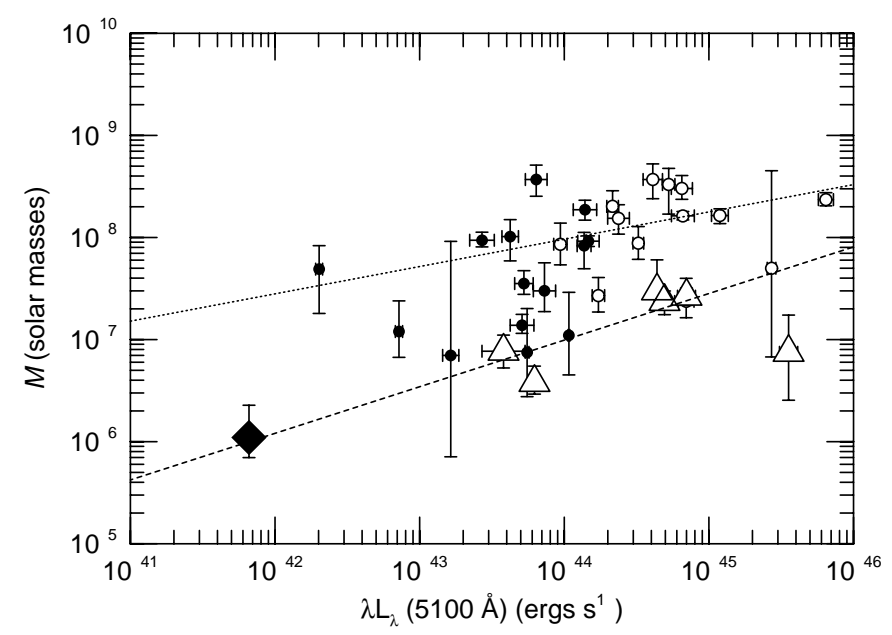

FIG. 7.--Relationship between the reverberation-based virial mass and optical luminosity for AGNs. Data points are coded as in Fig. 6. similar sources at intermediate inclination, the masses of low-inclination sources will be underestimated and their luminosities will be overestimated, displacing the narrowline objects in Figure 7 toward the lower right. This is generally consistent with the location of all seven of the narrow-line objects, including NGC 4051. The line transfer function for $\mathrm{H} \beta$ would provide a more definitive test of this hypothesis since it would allow determination of the inclination of the system. This would require more and better data than we have obtained in this experiment. However, as noted earlier, Christopoulou et al. (1997) show that the NLR morphology and kinematics suggest a system that is inclined to the line of sight by $\sim 50^{\circ}$. It seems reasonable to suppose that the NLR and BLR axes are approximately co-aligned. If this is the case, then our virial estimate for the central mass is too small by a modest factor of $\sin 50^{\circ} \approx 0.77$, and the corrected central mass is then $1.4_{-0.6}^{+1.0} \times 10^{6} M_{\odot}$.

3. Are NLS1s undermassive systems with relatively high accretion rates? Again, the distribution of the narrow-line objects, including NGC 4051, in Figure 7 is consistent with this hypothesis. The narrow-line sources on this plot lie below the mass-luminosity relationship for other AGNs, at the lower end of the envelope around this relationship.

In summary, the hypothesis that NLS1s have unusually distant BLRs for their luminosity is probably not viable in general, although it could apply to the specific case of NGC 4051. At the present time, however, we cannot distinguish between the low-inclination and low-mass, high-accretion rate hypotheses on the basis of the reverberation results alone. The latter is favored on the basis of X-ray considerations and the $50^{\circ}$ inclination inferred from the NLR. Indeed, it is entirely possible that both effects (i.e., low inclination and low black hole mass) contribute. An improvement in the optical spectroscopic monitoring data could allow determination of the $\mathrm{H} \beta$ transfer function, which could allow discrimination between these competing models.

\subsection{Broad He II Emission}

As noted in $\S 3.2$, we detected very broad, blueshifted He II $\lambda 4686$ emission in the rms spectra. The blueshift of this component is suggestive of radial rather than virialized motion. There is no similar obvious component to the Balmer lines, but we should expect that similar blueshifted features might appear in the other higher ionization lines in the UV. As no contemporaneous spectra were available, we retrieved the 31 International Ultraviolet Explorer (IUE) short-wavelength prime (SWP) camera (wavelength range $\sim 1150-2000 \AA$ A) spectra from the Space Telescope Science Institute Multimission Data Archive. We formed an average of all these spectra, since the individual spectra were of rather low signal-to-noise ratio. In Figure 8, we show the line profiles of He II $\lambda 4686$ (the rms profile, as in Fig. 5) and those of He II $\lambda 1640$ and C IV $\lambda 1549$ based on the mean IUE spectra. We note that each of these lines has a strong wing extending several thousand kilometers per second blueward of the line peak; indeed, the comparatively large widths and blueshifts of the UV lines in NLS1 spectra have been noted earlier by Rodríguez-Pascual, Mas-Hesse, \& Santos-Lleó (1997). It is possible that this gas is related to the known warm absorber in NGC 4051 (McHardy et al. 


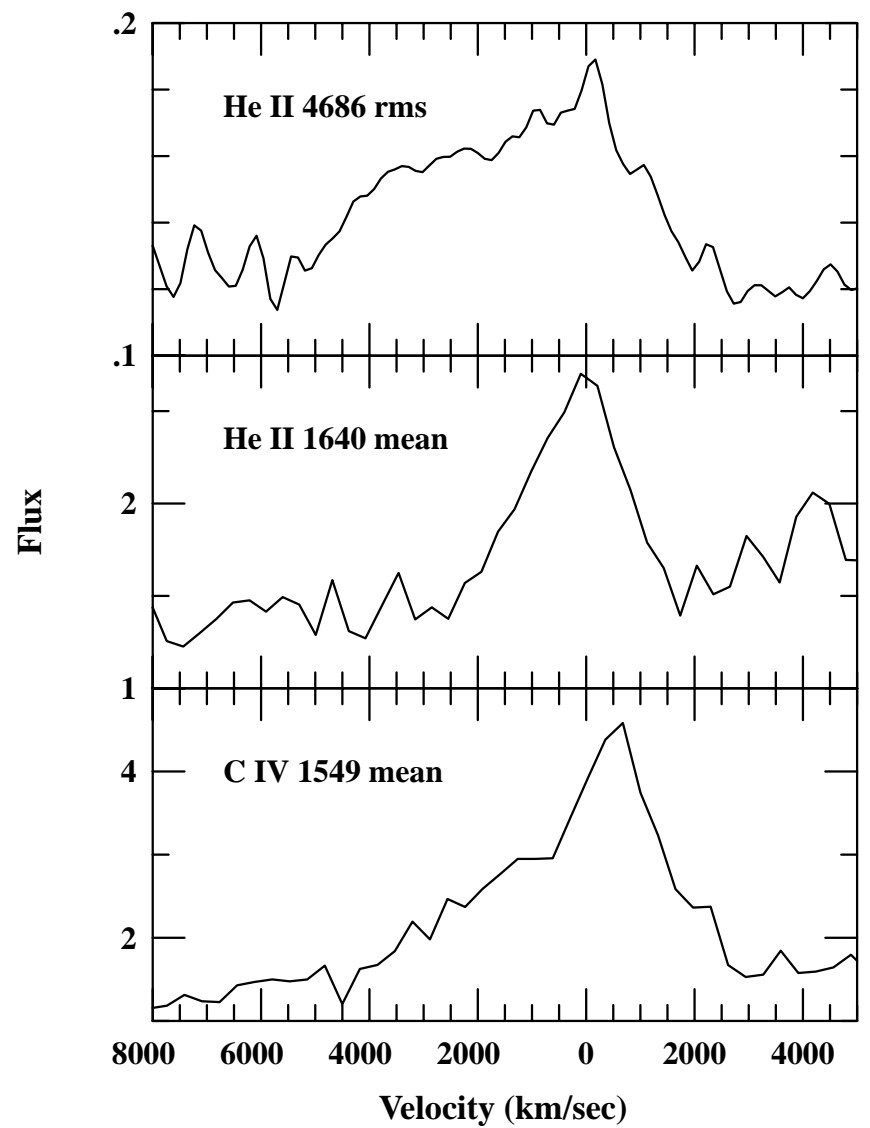

Fig. 8.-Top panel shows the profile of He II $\lambda 4686$ in the rms spectrum from year 1 (1996), as in Fig. 5. The profiles of He II $\lambda 1640$ and C IV $\lambda 1549$ from mean IUE spectra are shown in the middle and bottom panels, respectively. Fluxes are in units of $10^{-15} \mathrm{ergs} \mathrm{s}^{-1} \mathrm{~cm}^{-2} \AA^{-1}$, and in each panel the velocity scale is based on an assumed systemic redshift of $z=0.002418$ (de Vaucouleurs et al. 1991). The blue wing appears more pronounced in the top panel because the rms spectrum enhances the variable part of the line. Also, the optical and UV observations are not contemporaneous.

1995). Interestingly, photoionization equilibrium modeling of the X-ray warm absorber data (Nicastro et al. 1999) suggests a distance from the source of approximately 5 lt-days, which is consistent with the reverberation result given in Table 4.

The differences between the characteristics of the $\mathrm{H} \beta$ emission line on the one hand and the characteristics of the high-ionization lines on the other suggest a two-component BLR, which has been proposed on numerous occasions on other grounds (e.g., Collin-Souffrin et al. 1988; van Groningen 1987). In this particular case, an interpretation that is at least qualitatively consistent with all the data and relatively simple is that the Balmer lines arise primarily in material that is in a flattened disklike configuration at a low to moderate inclination (to account for the narrow width of the $\mathrm{H} \beta$ line), and the high-ionization lines arise in an outflowing wind, of which we see preferentially the component on the near side of the disk (to account for high velocity and blueward asymmetry). Such a model is illustrated schematically by Collin-Souffrin et al. (1988, their Fig. 1) and more recently by Dultzin-Hacyan, Taniguchi, \& Uranga (1999, their Fig. 1). This geometry was also suggested by van Groningen (1987) to explain the line profiles and profile ratios of the Balmer lines in Mrk 335, another narrow-line object.
As noted earlier, our virial mass estimate of $M=1.1 \times 10^{6} \quad M_{\odot}$ might seriously underestimate the black hole mass if the inclination of the system is very low. Moreover, some low-inclination accretion disk models predict relatively strong, variable $\mathrm{EUV} /$ soft X-ray fluxes (e.g., Netzer 1987; Madau 1988), consistent with observations of NLS1s. We therefore cannot rule out the possibility that the NLS1 phenomenon is due principally to inclination effects. However, the strong rapid X-ray variability of NLS1s seems to favor the low-mass, high accretion rate explanation, as does the $50^{\circ}$ inclination of the NLR, unless the BLR and NLR axes of symmetry are very different, which seems rather unlikely on physical grounds.

The behavior of the He II $\lambda 4686$ line during year 3 may provide additional information about the ionizing continuum at the time the X-rays went into an extremely faint state. In Figure 9, we show the rms spectrum based on set B spectra obtained between JD 2,450,810 and JD 2,451,022. The $\mathrm{H} \beta$ line is strong and narrow, as it is in the other rms spectra shown in Figure 5, which indicates that the continuum shortward of $912 \AA$ is still present and variable. However, the He II $\lambda 4686$ line is absent or very weak, indicating that the driving EUV continuum shortward of the He II edge at $228 \AA(54.4 \mathrm{eV})$ is not driving He II $\lambda 4686$ variations because the EUV flux is either low or varying little. Evidence from earlier years (Uttley et al. 1999) shows that the EUV and X-ray fluxes in NGC 4051 are well correlated, which implies that the EUV continuum might also have been extremely weak in year 3. Simultaneous BeppoSAX, RXTE, and Extreme Ultraviolet Explorer $(E U V E)$ observations obtained during the low state indicate that this is correct (Uttley et al. 2000). If the He II emission has dramatically decreased during year 3, we could infer that the transition radius between the inner ADAF region and the outer thin-disk structure must occur somewhere outside (or in the vicinity of) the region of the disk that contributes most of the flux at about $228 \AA$ but inside the radius that contributes most of the flux at about $912 \AA$.

In this regard, whether or not there is residual He II $\lambda 4686$ emission in the low state during year 3 becomes an interesting question. Note in particular that the measurements of the He II $\lambda 4686$ flux given in Table 2 and Figure 1 tell us little because these values include flux from broad-line $\mathrm{Fe}$ II blends and various narrow-line features. In order to address the question of how much $\mathrm{He}$ II $\lambda 4686$ persists in the low state, we have attempted a decomposition of the He II $\lambda 4686$ feature. Our first step was to form average high-state (as in the top panel of Fig. 5) and low-state spectra. We then attempted to remove the $\mathrm{Fe}$ II blends from the spectra by

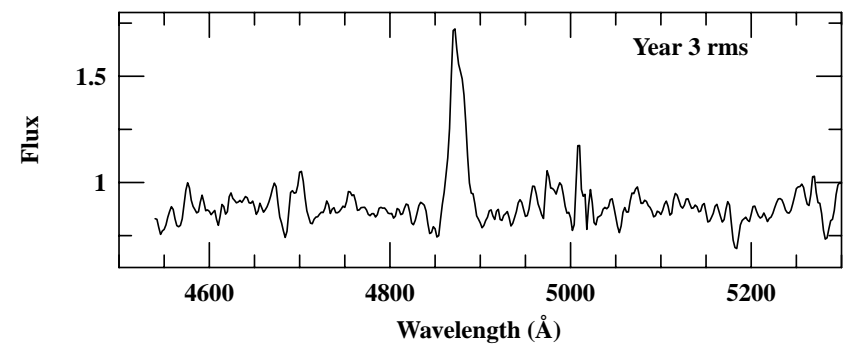

FIG. 9.- Shown is the rms spectrum based on the set B spectra obtained during year 3 between JD 2,450,810 and JD 2,451,022. Note that $\mathrm{H} \beta$ remains strongly variable, but in comparison to the rms spectra shown in Fig. 5, the He II $\lambda 4686$ line is much weaker, if present at all. 
using the optical Fe II template spectrum of Boroson \& Green (1992), convolved with a Gaussian to match the width of the features in the spectrum of NGC 4051. After subtraction of the flux-scaled and broadened Fe II template, we subtracted a power-law continuum from each spectrum. The He II $\lambda 4686$ region of the resulting high-state and lowstate spectra is shown in the top panel of Figure 10. We then subtracted the low-state spectrum from the high-state spectrum, forming the difference spectrum shown in the middle panel of Figure 10. The flux in the He II $\lambda 4686$ line in the difference spectrum is $7.4 \times 10^{-15} \mathrm{ergs} \mathrm{s}^{-1} \mathrm{~cm}^{-2}$. We then make the assumption that the $\mathrm{He}$ II line profile in the difference spectrum can be used to model the He II contribution in the low-state spectrum. We then scale the He II profile in flux on a trial-and-error basis and subtract it from the lowstate spectrum until the flux in the model profile causes the residual to have negative flux. Using this procedure, we find that the largest possible value for the $\mathrm{He}$ II $\lambda 4686$ line in the low state is $\sim 8.8 \times 10^{-15} \mathrm{ergs} \mathrm{s}^{-1} \mathrm{~cm}^{-2}$. These values mean that the He II $\lambda 4686$ flux decreased by at least $46 \%$ between the year 1 high state and the year 3 low state. Our conclusion is thus that while broad He II $\lambda 4686$ probably did not completely disappear during the low state in year 3 , the line flux did decrease dramatically and any $\mathrm{He}$ II variations during this period were too small to detect.

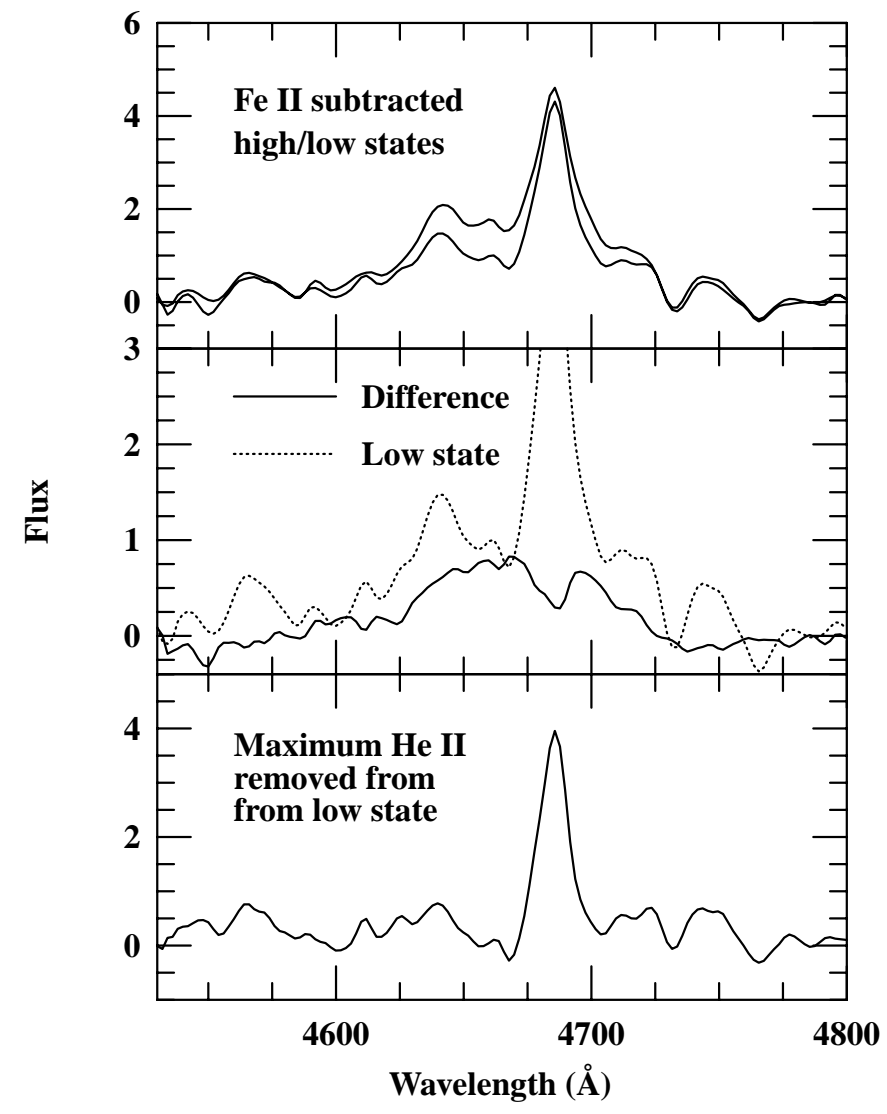

FIG. 10.-Top panel shows the He II $\lambda 4686$ region of the mean highstate and low-state spectra, with Fe II emission and the underlying continuum subtracted. The middle panel shows the difference between the high-state and low-state spectra. The low-state spectrum is shown for reference (note in particular the strong He II $\lambda 4686$ narrow-line component in the low-state spectrum). The bottom panel shows our best estimate of the low-state spectrum with the largest possible contribution of broad He II emission removed. Data are plotted in the rest frame of NGC 4051. The vertical axis is flux in units of $10^{-15} \mathrm{ergs} \mathrm{s}^{-1} \mathrm{~cm}^{-2} \AA^{-1}$.
In any case, these results underscore the importance of multiwavelength observations of NLS1s in very low states. Presumably, comparison of the strength of the various UV emission lines in the low state relative to the values in the high state could lead to determination of $r_{\text {tran }}$. Unfortunately, no ultraviolet data are available during the present campaign.

\section{SUMMARY}

On the basis of three years of combined X-ray and optical spectroscopic monitoring of the narrow-line Seyfert 1 galaxy NGC 4051, we reach the following conclusions:

1 . The rapid and strong X-ray variations that characterize narrow-line Seyfert 1 galaxies are detected in our X-ray observations of NGC 4051 but are not detected in the optical, consistent with previous findings.

2. On timescales of many weeks and longer, there does appear to be a correlation between the X-ray and optical continuum fluxes.

3. The variable part of the $\mathrm{H} \beta$ emission line has a Doppler width of $\sim 1100 \mathrm{~km} \mathrm{~s}^{-1}$ and a time delay relative to the continuum of about 6 days. Combining these quantities leads to a virial mass estimate of $\sim 1.1 \times 10^{6} M_{\odot}$. If we assume that the inclination of the system is $50^{\circ}$, as suggested by the NLR study of Christopoulou et al. (1997), then the mass of the central source is $\sim 1.4 \times 10^{6} M_{\odot}$.

4. The He II $\lambda 4686$ emission line is strongly variable, although an accurate time delay cannot be measured. This line is about 5 times as broad as the $\mathrm{H} \beta$ line and is strongly blueward asymmetric, as are the UV high-ionization lines in this object.

5. In the BLR radius-luminosity relationship, we find that narrow-line objects (those with $V_{\text {FWHM }} \lesssim 2000 \mathrm{~km} \mathrm{~s}^{-1}$ ) seem to fall on the same locus as AGNs with broad lines.

6. In the virial mass-luminosity relationship, narrow-line objects populate the low-mass end of a rather broad envelope; they have virial masses typically an order of magnitude lower than other AGNs of similar luminosity.

7. During the third year of this program, the hard X-ray flux decreased by approximately a factor of 10 , and the broad-line $\mathrm{He}$ II $\lambda 4686$ flux decreased by nearly a factor of 2 and did not show detectable variations during this low state. At the same time, the optical continuum and broad $\mathrm{H} \beta$ emission line were only slightly fainter than previously and continued to vary significantly. This suggests that the innermost part of the accretion disk went into an ADAF state, greatly reducing the production of high-energy continuum photons from the inner part of the accretion disk.

A picture that is consistent with the emission-line characteristics is one in which the Balmer lines arise primarily in a disklike configuration seen at low to moderate inclination and the high-ionization lines arise primarily in an outflowing wind. The high-ionization lines are blueward asymmetric because we see emission preferentially from the near side, with the far side at least partially obscured by the disk component (which might be an extension of the accretion disk). This geometry requires that much of the highionization line flux arises in a region of scale comparable to the disk system that emits the low-ionization lines and that the disk system is at least partially opaque to the He II line radiation. If NGC 4051 is typical of the NLS1 class, then it 
might be that NLS1s are best described as low-mass, highaccretion rate systems, although the possible role of inclination cannot be discounted. Indeed, the full explanation of the NLS1 phenomenon may involve both inclination and black hole mass.
For support of this work, we are grateful to the National Science Foundation (grant AST 94-20080 to Ohio State University). We thank the referee, M.-H. Ulrich, for suggestions that improved this paper.

\section{REFERENCES}

Blandford, R. D., \& McKee, C. F. 1982, ApJ, 255, 419

Boller, Th., Brandt, W. N., Fabian, A., \& Fink, H. 1997, MNRAS, 289, 393

Boller, Th., Brandt, W. N., \& Fink, H. 1996, A\&A, 305, 53

Boroson, T. A., \& Green, R. F. 1992, ApJS, 80, 109

Bradt, H. V. D., Rothschild, R. E. \& Swank, J. H. 1993, A\&AS, 97, 355

Brandt, W. N., Mathur, S., \& Elvis, M. 1997, MNRAS, 285, L25

Christopoulou, P. E., Holloway, A. J., Steffen, W., Mundell, C. G., Thean, A. H. C., Goudis, C. D., Meaburn, J., \& Pedlar, A. 1997, MNRAS, 284 385

Collin-Souffrin, S., Dyson, J. E., McDowell, J. C., \& Perry, J. J. 1988, MNRAS, 232, 539

Davidson, K., \& Kinman, T. D. 1978, ApJ, 225, 776

de Vaucouleurs, G., de Vaucouleurs, A., Corwin, H. G., Jr., Buta, R. J., Paturel, G., \& Fouqué, P. 1991, Third Reference Catalog of Bright Galaxies (New York: Springer)

Done, C., Ward, M. J., Fabian, A. C., Kunieda, H., Tsuruta, S., Lawrence, A., Smith, M. G., \& Wamsteker, W. 1990, MNRAS, 243, 713

Dultzin-Hacyan, D., Taniguchi, Y., \& Uranga, L. 1999, in Structure and Kinematics of Quasar Broad-Line Regions, ed. C. M. Gaskell, W. N. Brandt, D. Dultzin-Hacyan, M. Dietrich, \& M. Eracleous (San Francisco: ASP), 303

Edelson, R. A., \& Krolik, J. H. 1988, ApJ, 333, 646

Fabricant, D., Cheimets, P., Caldwell, J., \& Geary, J. 1998, PASP, 110, 79

Gaskell, C. M., \& Peterson, B. M. 1987, ApJS, 65, 1

Gaskell, C. M., \& Sparke, L. S. 1986, ApJ, 305, 175

Guilbert, P. W., \& Rees, M. J. 1988, MNRAS, 233, 475

Kaspi, S., Smith, P. S., Netzer, H., Maoz, D., Jannuzi, B. T., \& Giveon, U. 2000, ApJ, 533, 631

Lawrence, A., Watson, M. G., Pounds, K. A., \& Elvis, M. 1987, Nature, 325,694

Madau, P. 1988, ApJ, 327, 116

Maoz, D., Edelson, R., \& Nandra, K. 2000, AJ, 119, 119

Mason, K., Puchnarewicz, E. M., \& Jones, L. R. 1996, MNRAS, 283, L26

McHardy, I. M., Green, A. R., Done, C., Puchnarewicz, E. M., Mason, K. O., Branduardi-Raymont, G., \& Jones, M. H. 1995, MNRAS, 273, 549

Narayan, R., Mahadevan, R., Grindlay, J. E., Popham, R. G., \& Gammie, C. 1998, ApJ, 492, 554

Narayan, R., \& Yi, I. 1994, ApJ, 428, L13
Netzer, H. 1987, MNRAS, 225, 55

1990, in Active Galactic Nuclei, ed. T. J.-L. Courvoisier \& M. Mayor (Berlin: Springer), 141

Netzer, H., \& Peterson, B. M. 1997, in Astronomical Time Series, ed. D. Maoz, A. Sternberg, \& E. M. Liebowitz (Dordrecht: Kluwer), 85

Nicastro, F., Fiore, F., Perola, G. C., \& Elvis, M. 1999, ApJ, 512, 184

Orosz, J. A., Remillard, R. A., Bailyn, C. D., \& McClintock, J. E. 1997, ApJ, 478, L83

Osterbrock, D. E., \& Pogge, R. W. 1985, ApJ, 297, 166

Penston, M. V. 1991, in Variability of Galactic Nuclei, ed. H. R. Miller \& P. J. Wiita (Cambridge: Cambridge Univ. Press), 343

Peterson, B. M. 1993, PASP, 105, 247

Peterson, B. M., et al. 1999, ApJ, 510, 659

Peterson, B. M., \& Wandel, A. 1999, ApJ, 521, L95

Peterson, B. M., Wanders, I., Horne, K., Collier, S., Alexander, T., Kaspi, S., \& Maoz, D. 1998, PASP, 110, 660

Puchnarewicz, E. M., Mason, K. O., Córdova, F. A., Kartje, J., Branduardi-Raymont, G., Mittaz, J. P. D., Murdin, P. G., \& AllingtonSmith, J. 1992, MNRAS, 256, 589

Rodríguez-Pascual, P. M., et al. 1997, ApJ, 110, 9

Rodríguez-Pascual, P. M., Mas-Hesse, J. M., \& Santos-Lleó, M. 1997, $\mathrm{A} \& \mathrm{~A}, 327,72$

Seyfert, C. 1943, ApJ, 97, 28

Shakura, R. I., \& Sunyaev, R. A. 1973, A\&A, 24, 337

Shields, J. C., Ferland, G. J., \& Peterson, B. M. 1995, ApJ, 441, 507

Taniguchi, Y., Murayama, T., \& Nagao, T. 2000, ApJ, submitted (astro$\mathrm{ph} / 9910036)$

Uttley, P., McHardy, I. M., Papadakis, I. E., Cagnoni, F., \& Fruscione, A. 2000, MNRAS, 312, 880

Uttley, P., McHardy, I. M., Papadakis, I. E., Guainazzi, M., \& Fruscione, A. 1999, MNRAS, 307, L6

van Groningen, E. 1987, A\&A, 186, 103

van Groningen, E., \& Wanders, I. 1992, PASP, 104, 700

Wandel, A., \& Boller, Th. 1998, A\&A, 331, 884

Wandel, A., Peterson, B. M., \& Malkan, M. A. 1999, ApJ, 526, 579

White, R. J., \& Peterson, B. M. 1994, PASP, 106, 879

Young, A. J., Crawford, C. S., Fabian, A. C., Brandt, W. N., \& O'Brien, P. T. 1999, MNRAS, 304, L46 